\title{
DOUBLE-BARRIER PARISIAN OPTIONS
}

\author{
ANGELOS DASSIOS *** AND \\ SHANLE WU, ${ }^{*}$ London School of Economics
}

\begin{abstract}
In this paper we study the excursion time of a Brownian motion with drift outside a corridor by using a four-state semi-Markov model. In mathematical finance, these results have an important application in the valuation of double-barrier Parisian options. We subsequently obtain an explicit expression for the Laplace transform of its price.
\end{abstract}

Keywords: Excursion time; four-state semi-Markov model; double-barrier Parisian option; Laplace transform

2010 Mathematics Subject Classification: Primary 91B28

Secondary 60J65; 60G44; 60J25

\section{Introduction}

The concept of Parisian options was first introduced by Chesney et al. [4]. It is a special case of path-dependent options. The owner of a Parisian option will either gain the right or lose the right to exercise the option upon the price reaching a predetermined barrier level $L$ and staying above or below the level for a predetermined time $d$ before the maturity date $T$.

More precisely, the owner of a Parisian down-and-out option loses the option if the underlying asset price $S$ reaches the level $L$ and remains constantly below this level for a time interval longer than $d$. For a Parisian down-and-in option, the same event gives the owner the right to exercise the option. For details on the pricing of Parisian options, see [4], [8], and [13].

Double-barrier Parisian options are a two-barrier version of the standard Parisian options introduced by Chesney et al. [4]. In contrast to the Parisian options mentioned above, we consider the excursions both below the lower barrier and above the upper barrier, i.e. outside a corridor formed by these two barriers. Let us look at two examples, depending on whether the condition is that the required excursions above the upper barrier and below the lower barrier have to both happen before the maturity date or that either one of them happens before the maturity. In the first example, the owner of a double-barrier Parisian max-out option loses the option if the underlying asset price process $S$ has both an excursion above the upper barrier for longer than a continuous period $d_{1}$ and below the lower barrier for longer than $d_{2}$ before the maturity of the option. In the second example, the owner of a double-barrier Parisian min-out option loses the right to exercise the option if either one of these two events happens before the maturity. Later on, we will derive the Laplace transforms which can be used to price options of this type.

In this paper we are going to use the same definition for the excursion as in [4] and [5]. Let $S$ be a stochastic process, and let $l_{1}$ and $l_{2}, l_{1}>l_{2}$, be the levels of these two barriers. As in [4],

Received 15 April 2008; revision received 25 August 2010.

* Postal address: Department of Statistics, London School of Economics, Houghton Street, London WC2A 2AE, UK.

** Email address: a.dassios@1se.ac.uk 
we define

$$
g_{l_{i}, t}^{S}:=\sup \left\{s \leq t \mid S_{S}=l_{i}\right\}, \quad d_{l_{i}, t}^{S}:=\inf \left\{s \geq t \mid S_{s}=l_{i}\right\}, \quad i=1,2,
$$

with the usual conventions that $\sup \{\varnothing\}=0$ and $\inf \{\varnothing\}=\infty$. Assuming that $d_{1}>0$ and $d_{2}>0$, we now define

$$
\begin{aligned}
\tau_{1}^{S} & :=\inf \left\{t>0 \mid \mathbf{1}_{\left\{S_{t}>l_{1}\right\}}\left(t-g_{l_{1}, t}^{S}\right) \geq d_{1}\right\}, \\
\tau_{2}^{S} & :=\inf \left\{t>0 \mid \mathbf{1}_{\left\{l_{2}<S_{t}<l_{1}\right\}} \mathbf{1}_{\left\{g_{l_{1}, t}^{S}>g_{l_{2}, t}^{S}\right\}}\left(t-g_{l_{1}, t}^{S}\right) \geq d_{2}\right\}, \\
\tau_{3}^{S} & :=\inf \left\{t>0 \mid \mathbf{1}_{\left\{l_{2}<S_{t}<l_{1}\right\}} \mathbf{1}_{\left\{g_{l_{1}, t}^{S}<g_{l_{2}, t}^{S}\right\}}\left(t-g_{l_{2}, t}^{S}\right) \geq d_{3}\right\}, \\
\tau_{4}^{S} & :=\inf \left\{t>0 \mid \mathbf{1}_{\left\{S_{t}<l_{2}\right\}}\left(t-g_{l_{2}, t}^{S}\right) \geq d_{4}\right\}, \\
\tau^{S} & :=\tau_{1}^{S} \wedge \tau_{4}^{S} .
\end{aligned}
$$

We can see that $\tau_{1}^{S}$ is the first time that the length of the excursion of the process $S$ above the barrier $l_{1}$ reaches a given level $d_{1}, \tau_{4}^{S}$ corresponds to the one below $l_{2}$ with required length $d_{4}$, and $\tau^{S}$ is the smaller of $\tau_{1}^{S}$ and $\tau_{4}^{S}$. We also see that $\tau_{2}^{S}$ is the first time that the length of the excursion in the corridor reaches given level $d_{2}$, given that the excursion starts from the upper barrier $l_{1}$; and $\tau_{3}^{S}$ corresponds to the one in the corridor starting from the lower barrier $l_{2}$. Our aim is to study the excursion outside the corridor; therefore, $\tau_{2}^{S}$ and $\tau_{3}^{S}$ are not of interest here. However, we need to use these two stopping times to define our four-state semi-Markov model that will be the main tool used for calculation.

Now assume that $r$ is the risk-free rate, $T$ is the term of the option, $S_{t}$ is the price of its underlying asset, $K$ is the strike price, and $Q$ is the risk neutral measure. If we have a double-barrier Parisian min-out call option with barriers $l_{1}$ and $l_{2}$, its price can be expressed as

$$
\mathrm{DP}_{\text {min-out call }}=\mathrm{e}^{-r T} \mathrm{E}_{Q}\left(\mathbf{1}_{\left\{\tau^{S}>T\right\}}\left(S_{T}-K\right)^{+}\right),
$$

and the price of a double-barrier Parisian min-in put option is expressed as

$$
\mathrm{DP}_{\text {min-in put }}=\mathrm{e}^{-r T} \mathrm{E}_{Q}\left(\mathbf{1}_{\{\tau} \tau_{<T\}}\left(K-S_{T}\right)^{+}\right) \text {. }
$$

In this paper we study the excursion time outside the corridor using a semi-Markov model consisting of four states. By applying the model to a Brownian motion we can obtain the explicit form of the Laplace transform for the price of double-barrier options. We can then invert using techniques given in [8].

In Section 2 we introduce the four-state semi-Markov model as well as a new process, the doubly perturbed Brownian motion, which has the same behaviour as a Brownian motion except that each time it hits one of the two barriers, it moves towards the other side of the barrier by a jump of size $\varepsilon$. In Section 3 we obtain the martingale to which we can apply the optional sampling theorem and obtain the Laplace transform that we can use for pricing later. We give our main results applied to Brownian motion in Section 4, including the Laplace transforms for the stopping times we defined in (1)-(5) for both a Brownian motion with drift, i.e. $S=W^{\mu}$, and a standard Brownian motion, i.e. $S=W$. In Section 5 we focus on pricing the double-barrier Parisian options.

\section{Definitions}

From the description above, it is clear that we are actually considering four states: the state when the stochastic process is above the barrier $l_{1}$; the state when it is below $l_{2}$; and two states 
when it is between $l_{1}$ and $l_{2}$, depending on whether it comes into the corridor through $l_{1}$ or $l_{2}$. For each state, we are interested in the time the process spends in it. We introduce a new process:

$$
Z_{t}^{S}:= \begin{cases}1 & \text { if } S_{t}>l_{1}, \\ 2 & \text { if } l_{1}>S_{t}>l_{2} \text { and } g_{l_{1}, t}^{S}>g_{l_{2}, t}^{S}, \\ 3 & \text { if } l_{1}>S_{t}>l_{2} \text { and } g_{l_{1}, t}^{S}<g_{l_{2}, t}^{S}, \\ 4 & \text { if } S_{t}<l_{2} .\end{cases}
$$

We can now express the variables defined above in terms of $Z_{t}$ :

$$
\begin{aligned}
g_{l_{i}, t}^{S} & =\sup \left\{s \leq t \mid Z_{s}^{S} \neq Z_{t}\right\}, \\
d_{l_{i}, t}^{S} & =\inf \left\{s \geq t \mid Z_{s}^{S} \neq Z_{t}\right\}, \\
\tau_{1}^{S} & =\inf \left\{t>0 \mid \mathbf{1}_{\left\{Z_{t}^{S}=1\right\}}\left(t-g_{l_{1}, t}^{S}\right) \geq d_{1}\right\}, \\
\tau_{2}^{S} & =\inf \left\{t>0 \mid \mathbf{1}_{\left\{Z_{t}^{S}=2\right\}}\left(t-g_{l_{1}, t}^{S}\right) \geq d_{2}\right\}, \\
\tau_{3}^{S} & =\inf \left\{t>0 \mid \mathbf{1}_{\left\{Z_{t}^{S}=3\right\}}\left(t-g_{l_{2}, t}^{S}\right) \geq d_{3}\right\}, \\
\tau_{4}^{S} & =\inf \left\{t>0 \mid \mathbf{1}_{\left\{Z_{t}^{S}=4\right\}}\left(t-g_{l_{2}, t}^{S}\right) \geq d_{4}\right\} .
\end{aligned}
$$

We then define

$$
V_{t}^{S}:=t-\max \left(g_{l_{1}, t}^{S}, g_{l_{2}, t}^{S}\right),
$$

the time $Z_{t}^{S}$ has spent in the current state. It is easy to see that $\left(Z_{t}^{S}, V_{t}^{S}\right)$ is a Markov process. Therefore, $Z_{t}^{S}$ is a semi-Markov process with state space $\{1,2,3,4\}$, where 1 stands for the state when the stochastic process $S$ is above the barrier $l_{1}, 4$ corresponds to the state below the barrier $l_{2}$, and 2 and 3 represent the states when $S$ is in the corridor given that it comes in through $l_{1}$ and $l_{2}$, respectively.

For $Z_{t}^{S}$, the transition intensities $\lambda_{i j}(u)$ satisfy

$$
\begin{aligned}
& \mathrm{P}\left(Z_{t+\Delta t}^{S}=j, i \neq j \mid Z_{t}^{S}=i, V_{t}^{S}=u\right)=\lambda_{i j}(u) \Delta t+o(\Delta t), \\
& \mathrm{P}\left(Z_{t+\Delta t}^{S}=i \mid Z_{t}^{S}=i, V_{t}^{S}=u\right)=1-\sum_{i \neq j} \lambda_{i j}(u) \Delta t+o(\Delta t) .
\end{aligned}
$$

Define

$$
\bar{P}_{i}(\mu):=\exp \left\{-\int_{0}^{\mu} \sum_{i \neq j} \lambda_{i j}(v) \mathrm{d} v\right\}, \quad p_{i j}(\mu)=\lambda_{i j}(\mu) \bar{P}_{i}(\mu) .
$$

Note that

$$
P_{i}(\mu)=1-\bar{P}_{i}(\mu)
$$

is the distribution function of the excursion time in state $i$, which is a random variable $U_{i}$ defined as

$$
U_{i}:=\inf _{s>0}\left\{Z_{s}^{S} \neq i \mid Z_{0}^{S}=i, V_{0}^{S}=0\right\} .
$$

Note that because the process is time homogeneous, this has the same distribution as

$$
\inf _{s>0}\left\{Z_{t+s}^{S} \neq i \mid Z_{t}^{S}=i, V_{t}^{S}=0\right\} \quad \text { for any time } t .
$$


We therefore have

$$
p_{i j}(\mu)=\lim _{\Delta \mu \rightarrow 0} \frac{\mathrm{P}\left(U_{i} \in(\mu, \mu+\Delta \mu), Z_{U_{i}}^{S}=j\right)}{\Delta \mu} .
$$

Moreover, in the definition of $Z^{S}$, we deliberately ignore the situation when $S_{t}=l_{i}, i=1,2$. The reason is that we consider only the processes for which $\int_{0}^{t} \mathbf{1}_{\left\{S_{u}=l_{i}\right\}} \mathrm{d} u=0, i=1,2$.

Also, when $l_{1}$ and $l_{2}$ are regular points of the process (see [1] for a definition), we have to deal with the degeneration of $p_{i j}$. Let us take a Brownian motion as an example. Assume that $W_{t}^{\mu}=\mu t+W_{t}$ with $\mu \geq 0$, where $W_{t}$ is a standard Brownian motion. Setting $x_{0}$ to be its starting point, we know that its density for the first hitting time of level $l_{i}, i=1,2$, is

$$
p_{x_{0}}=\frac{\left|l_{i}-x_{0}\right|}{\sqrt{2 \pi t^{3}}} \exp \left\{-\frac{\left(l_{i}-x_{0}-\mu t\right)^{2}}{2 t}\right\}
$$

(see [12, Sections I.9 and I.13]). According to the definition of the transition density, $p_{12}(t)=$ $p_{21}(t)=p_{l_{1}}(t)=0$ and $p_{34}(t)=p_{43}(t)=p_{l_{2}}(t)=0$ for $t>0$.

The problem is not regularity in itself, but the fact that there are infinitely many excursions outside and also inside the barriers. In [6], in order to solve the single-barrier problem, we introduced the perturbed Brownian motion $X_{t}^{(\varepsilon)}$ with respect to the barrier we are interested in. We will extend this idea here, and construct a new process, double-perturbed Brownian motion. The anonymous referee pointed out that maybe regularity itself should be exploited in an attempt to considerably simplify our proofs (using perhaps an approach as in [9, Section III.2]). Moreover, the referee also suggested the use of excursion theory as in [10] and [11, Section VI.8]. This approach seems suitable for simplifying the arguments in [6]; a similar promising line can be found in [14, Chapter 15], where the excursion time is formulated as a Markov process whose generator is provided. However, there are two reasons why we will not adopt these ideas here. One reason is that our method can also be used to generalise some of our results for Lévy processes that can have jumps. The most important reason is that we make use of excursions between the two barriers. These are not discussed in the references mentioned and so our method seems the most appropriate one at this stage.

We now construct the new process, double-perturbed Brownian motion, $Y_{t}^{(\varepsilon)}, \varepsilon>0$, with respect to barriers $l_{1}$ and $l_{2}$. Assume that $W_{0}^{\mu}=l_{1}+\varepsilon$. Define the sequence of stopping times

$$
\delta_{0}=0, \quad \sigma_{n}=\inf \left\{t>\delta_{n} \mid W_{t}^{\mu}=l_{1}\right\}, \quad \delta_{n+1}=\inf \left\{t>\sigma_{n} \mid W_{t}^{\mu}=l_{1}+\varepsilon\right\},
$$

where $n=0,1, \ldots$ (see Figure 1$)$. Now define

$$
X_{t}^{(\varepsilon)}:= \begin{cases}W_{t}^{\mu} & \text { if } \delta_{n} \leq t<\sigma_{n}, \\ W_{t}^{\mu}-\varepsilon & \text { if } \sigma_{n} \leq t<\delta_{n+1}\end{cases}
$$

Similarly, we now define another sequence of stopping times with respect to the process $X_{t}^{(\varepsilon)}$ and barrier $l_{2}$ :

$$
\zeta_{0}:=0, \quad \eta_{n}:=\inf \left\{t>\zeta_{n} \mid X_{t}^{(\varepsilon)}=l_{2}\right\}, \quad \zeta_{n+1}:=\inf \left\{t>\eta_{n} \mid X_{t}^{(\varepsilon)}=l_{2}+\varepsilon\right\},
$$

where $n=0,1, \ldots$ (see Figure 2). Now define

$$
Y_{t}^{(\varepsilon)}:= \begin{cases}X_{t}^{(\varepsilon)} & \text { if } \zeta_{n} \leq t<\eta_{n} \\ X_{t}^{(\varepsilon)}-\varepsilon & \text { if } \eta_{n} \leq t<\zeta_{n+1}\end{cases}
$$




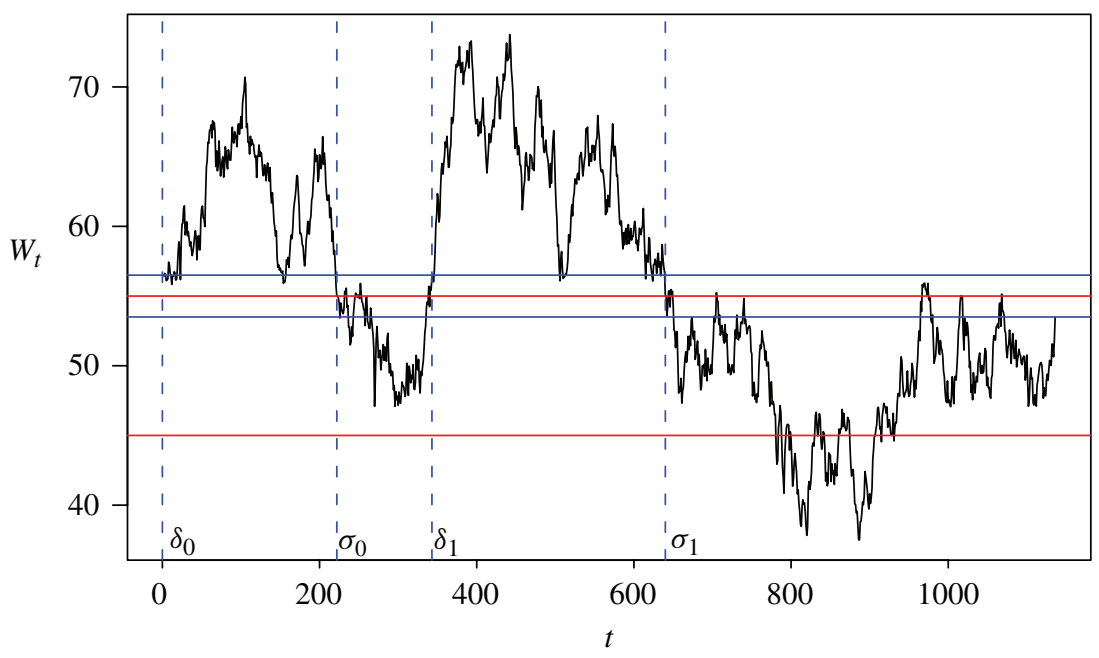

FIGURE 1: A sample path of the original Brownian motion, $W_{t}^{(\varepsilon)}$.

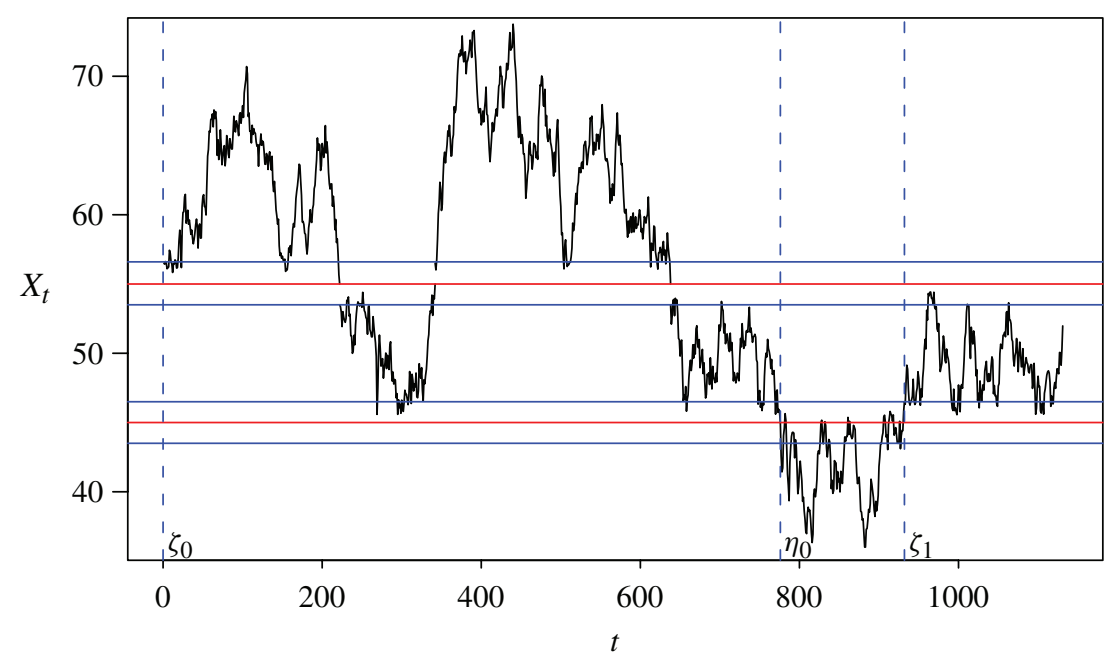

Figure 2: A sample path of the process $X_{t}^{(\varepsilon)}$.

It is actually a process which starts from $l_{1}+\varepsilon$ and has the same behaviour as the related Brownian motion, except that each time it hits the barrier $l_{1}$ or $l_{2}$, it will jump towards the opposite side of the barrier with size $\varepsilon$ (see Figure 3).

From the definition, it is clear that $l_{1}$ and $l_{2}$ become irregular points for $Y_{t}^{(\varepsilon)}$. Also, $Y_{t}^{(\varepsilon)}$ converges to $W_{t}^{\mu}$ with $W_{0}^{\mu}=l_{1}$ almost surely for all $t$. Therefore, as we prove in Appendix A, the Laplace transforms of the variables defined based on $Y_{t}^{(\varepsilon)}$ converge to those based on $W_{t}^{\mu}$. As a result, we can obtain the results for the Brownian motion by carrying out the calculation for $Y_{t}^{(\varepsilon)}$ and taking the limit $\varepsilon \rightarrow 0$. 


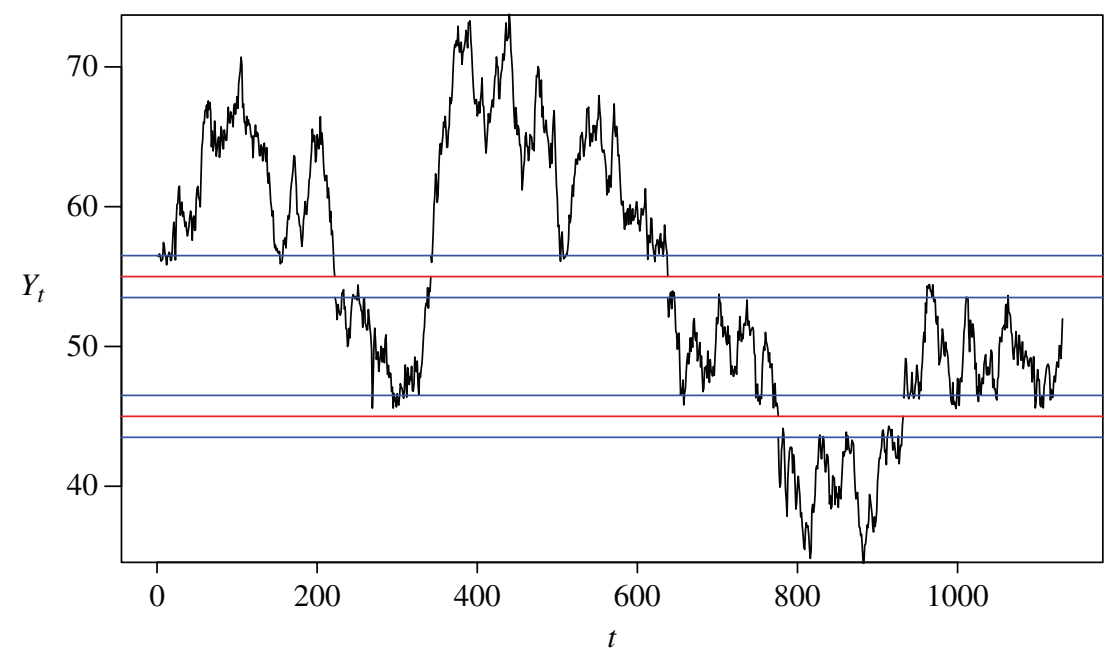

FIGURE 3: A sample path of the process $Y_{t}^{(\varepsilon)}$.

For $Y_{t}^{(\varepsilon)}$, we can define $Z^{Y}, \tau_{1}^{Y}, \tau_{2}^{Y}$, and $\tau^{Y}$ as above (we suppress the ( $\varepsilon$ ) superscript). For $Z^{Y}$, we have the transition densities (see [2, Equations 2.0.2 and 3.0.6])

$$
\begin{aligned}
& p_{12}(t)=\frac{\varepsilon}{\sqrt{2 \pi t^{3}}} \exp \left\{-\frac{(\varepsilon+\mu t)^{2}}{2 t}\right\} \\
& p_{21}(t)=\exp \left\{\mu \varepsilon-\frac{\mu^{2} t}{2}\right\} s s_{t}\left(l_{1}-l_{2}-\varepsilon, l_{1}-l_{2}\right), \\
& p_{24}(t)=\exp \left\{-\mu\left(l_{1}-l_{2}-\varepsilon\right)-\frac{\mu^{2} t}{2}\right\} s s_{t}\left(\varepsilon, l_{1}-l_{2}\right), \\
& p_{31}(t)=\exp \left\{\mu\left(l_{1}-l_{2}-\varepsilon\right)-\frac{\mu^{2} t}{2}\right\} s s_{t}\left(\varepsilon, l_{1}-l_{2}\right), \\
& p_{34}(t)=\exp \left\{-\mu \varepsilon-\frac{\mu^{2} t}{2}\right\} s s_{t}\left(l_{1}-l_{2}-\varepsilon, l_{1}-l_{2}\right), \\
& p_{43}(t)=\frac{\varepsilon}{\sqrt{2 \pi t^{3}}} \exp \left\{-\frac{(\varepsilon-\mu t)^{2}}{2 t}\right\}
\end{aligned}
$$

where

$$
s s_{t}(x, y)=\sum_{k=-\infty}^{\infty} \frac{(2 k+1) y-x}{\sqrt{2 \pi t^{3}}} \exp \left\{-\frac{((2 k+1) y-x)^{2}}{2 t}\right\} .
$$

Also, we know that

$$
p_{23}(t)=p_{32}(t)=p_{14}(t)=p_{41}(t)=0 .
$$

Clearly, all the arguments above apply to the standard Brownian motion, which is a special case of $W_{t}^{\mu}$ when $\mu=0$. 


\section{Results for the semi-Markov model}

In Section 2 we introduced the Markov process $\left(Z_{t}^{S}, V_{t}^{S}\right)$. Now we apply the same definition to the doubly perturbed Brownian motion $Y_{t}^{(\varepsilon)}$; therefore, we have $\left(Z_{t}^{Y}, V_{t}^{Y}\right)$, where $Z_{t}^{Y}$ is the current state of $Y_{t}^{(\varepsilon)}$, taking values from the state space $\{1,2,3,4\}$, and $V_{t}^{Y}$ is the time $Y_{t}^{(\varepsilon)}$ has spent in the current state. The time $V_{t}^{Y}$ is also a stochastic process. Now we consider a function of the form

$$
f\left(V_{t}^{Y}, Z_{t}^{Y}, t\right)=f_{Z_{t}^{Y}}\left(V_{t}^{Y}, t\right)
$$

where the $f_{i}, i=1,2,3,4$, are functions from $\mathbb{R}^{2}$ to $\mathbb{R}$. The generator $\mathcal{A}$ is defined as an operator such that

$$
f\left(V_{t}^{Y}, Z_{t}^{Y}, t\right)-\int_{0}^{s} \mathcal{A} f\left(V_{s}^{Y}, Z_{s}^{Y}, s\right) \mathrm{d} s
$$

is a martingale (see [7, Chapter 2]). Therefore, solving

$$
\mathcal{A} f=0
$$

subject to certain conditions will provide us with martingales of the form $f\left(V_{t}^{Y}, Z_{t}^{Y}, t\right)$, to which we can apply the optional stopping theorem to obtain the Laplace transform we are interested in. More precisely, we will have

$$
\begin{aligned}
\mathcal{A} f_{1}(u, t)= & \frac{\partial f_{1}(u, t)}{\partial t}+\frac{\partial f_{1}(u, t)}{\partial u}+\lambda_{12}(u)\left(f_{2}(0, t)-f_{1}(u, t)\right), \\
\mathcal{A} f_{2}(u, t)= & \frac{\partial f_{2}(u, t)}{\partial t}+\frac{\partial f_{2}(u, t)}{\partial u}+\lambda_{21}(u)\left(f_{1}(0, t)-f_{2}(u, t)\right) \\
& +\lambda_{24}(u)\left(f_{4}(0, t)-f_{2}(u, t)\right), \\
\mathcal{A} f_{3}(u, t)= & \frac{\partial f_{3}(u, t)}{\partial t}+\frac{\partial f_{3}(u, t)}{\partial u}+\lambda_{31}(u)\left(f_{1}(0, t)-f_{3}(u, t)\right) \\
& +\lambda_{34}(u)\left(f_{4}(0, t)-f_{3}(u, t)\right), \\
\mathcal{A} f_{4}(u, t)= & \frac{\partial f_{4}(u, t)}{\partial t}+\frac{\partial f_{4}(u, t)}{\partial u}+\lambda_{43}(u)\left(f_{4}(0, t)-f_{3}(u, t)\right) .
\end{aligned}
$$

Assume that $f_{i}$ has the form

$$
f_{i}(u, t)=\mathrm{e}^{-\beta t} g_{i}(u) .
$$

By solving the equation $\mathcal{A} f=0$, i.e.

$$
\mathcal{A} f_{1}=0, \quad \mathcal{A} f_{2}=0, \quad \mathcal{A} f_{3}=0, \quad \mathcal{A} f_{4}=0
$$

subject to

$$
g_{1}\left(d_{1}\right)=\alpha_{1}, \quad g_{2}\left(d_{2}\right)=\alpha_{2}, \quad g_{3}\left(d_{2}\right)=\alpha_{3}, \quad g_{4}\left(d_{2}\right)=\alpha_{4},
$$

we obtain

$$
\begin{aligned}
g_{i}(u)= & \alpha_{i} \exp \left\{-\int_{u}^{d_{i}}\left(\beta+\sum_{j \neq i} \lambda_{i j}(v)\right) \mathrm{d} v\right\} \\
& +\sum_{j \neq i} g_{j}(0) \int_{u}^{d_{i}} \lambda_{i j}(s) \exp \left\{-\int_{u}^{s}\left(\beta+\sum_{k \neq i} \lambda_{i k}(v)\right) \mathrm{d} v\right\} \mathrm{d} s .
\end{aligned}
$$


In our case, we are interested only in the excursion outside the corridor. Hence, we set $d_{2}$ and $d_{3}$ to be $\infty$. Also, $\lim _{d_{2} \rightarrow \infty} g_{2}\left(d_{2}\right)=\lim _{d_{3} \rightarrow \infty} g_{3}\left(d_{3}\right)=0$ gives $\alpha_{2}=\alpha_{3}=0$. Therefore, we have

$$
\begin{aligned}
& g_{1}(0)=\alpha_{1} \mathrm{e}^{-\beta d_{1}} \bar{P}_{1}\left(d_{1}\right)+\left\{g_{1}(0) \hat{P}_{21}(\beta)+g_{4}(0) \hat{P}_{24}(\beta)\right\} \tilde{P}_{12}(\beta), \\
& g_{4}(0)=\alpha_{4} \mathrm{e}^{-\beta d_{4}} \bar{P}_{4}\left(d_{4}\right)+\left\{g_{1}(0) \hat{P}_{31}(\beta)+g_{4}(0) \hat{P}_{34}(\beta)\right\} \tilde{P}_{43}(\beta) .
\end{aligned}
$$

Solving (14) and (15) gives

$$
\begin{aligned}
g_{1}(0)= & {\left[\alpha_{1} \exp \left\{\beta d_{1}\right\} \bar{P}_{1}\left(d_{1}\right)\left(1-\hat{P}_{34}(\beta) \tilde{P}_{43}(\beta)\right)+\alpha_{4} \exp \left\{\beta d_{4}\right\} \bar{P}_{4}\left(d_{4}\right) \hat{P}_{24}(\beta) \tilde{P}_{12}(\beta)\right] } \\
& \times\left[1-\hat{P}_{21}(\beta) \tilde{P}_{12}(\beta)-\hat{P}_{34}(\beta) \tilde{P}_{43}(\beta)+\hat{P}_{21}(\beta) \tilde{P}_{12}(\beta) \hat{P}_{34}(\beta) \tilde{P}_{43}(\beta)\right. \\
& \left.\quad-\hat{P}_{31}(\beta) \tilde{P}_{43}(\beta) \hat{P}_{24}(\beta) \tilde{P}_{12}(\beta)\right]^{-1}, \\
g_{4}(0)= & {\left[\alpha_{4} \exp \left\{\beta d_{4}\right\} \bar{P}_{4}\left(d_{4}\right)\left(1-\hat{P}_{21}(\beta) \tilde{P}_{12}(\beta)\right)+\alpha_{1} \exp \left\{\beta d_{1}\right\} \bar{P}_{1}\left(d_{1}\right) \hat{P}_{31}(\beta) \tilde{P}_{43}(\beta)\right] } \\
& \times\left[1-\hat{P}_{21}(\beta) \tilde{P}_{12}(\beta)-\hat{P}_{34}(\beta) \tilde{P}_{43}(\beta)+\hat{P}_{21}(\beta) \tilde{P}_{12}(\beta) \hat{P}_{34}(\beta) \tilde{P}_{43}(\beta)\right. \\
& \left.\quad-\hat{P}_{31}(\beta) \tilde{P}_{43}(\beta) \hat{P}_{24}(\beta) \tilde{P}_{12}(\beta)\right]^{-1},
\end{aligned}
$$

where

$$
\begin{aligned}
& \hat{P}_{i j}(\beta)=\int_{0}^{\infty} \mathrm{e}^{-\beta s} p_{i j}(s) \mathrm{d} s, \\
& \tilde{P}_{i j}(\beta)=\int_{0}^{d_{i}} \mathrm{e}^{-\beta s} p_{i j}(s) \mathrm{d} s .
\end{aligned}
$$

As a result, we have obtained the martingale

$$
M_{t}=f\left(V_{t}^{Y}, t\right)=\mathrm{e}^{-\beta t} g_{Z_{t}^{Y}}\left(V_{t}^{Y}\right), \quad i=1,2,3,4 .
$$

We now can apply the optional stopping theorem to $M_{t}$ with the stopping time $\tau^{Y} \wedge t$, where $\tau^{Y}$ is the stopping time defined by (5):

$$
\mathrm{E}\left(M_{\tau^{Y} \wedge t}\right)=\mathrm{E}\left(M_{0}\right) .
$$

The right-hand side of (16) is

$$
\mathrm{E}\left(M_{\tau^{Y} \wedge t}\right)=\mathrm{E}\left(M_{\tau^{Y}} \mathbf{1}_{\left\{\tau^{Y}<t\right\}}\right)+\mathrm{E}\left(M_{t} \mathbf{1}_{\left\{\tau^{Y}>t\right\}}\right) .
$$

Furthermore,

$$
\begin{aligned}
& \mathrm{E}\left(M_{\tau^{Y}} \mathbf{1}_{\left\{\tau^{Y}<t\right\}}\right) \\
&= \mathrm{E}\left(M_{\tau^{Y}} \mathbf{1}_{\left\{\tau_{1}^{Y}<\tau_{4}^{Y}\right\}} \mathbf{1}_{\left\{\tau_{1}^{Y}<t\right\}}\right)+\mathrm{E}\left(M_{\tau^{Y}} \mathbf{1}_{\left\{\tau_{1}^{Y}>\tau_{4}^{Y}\right\}} \mathbf{1}_{\left\{\tau_{4}^{Y}<t\right\}}\right) \\
&= \mathrm{E}\left(\exp \left\{-\beta \tau^{Y}\right\} g_{1}\left(d_{1}\right) \mathbf{1}_{\left\{\tau_{1}^{Y}<\tau_{4}^{Y}\right\}} \mathbf{1}_{\left\{\tau_{1}^{Y}<t\right\}}\right) \\
&+\mathrm{E}\left(\exp \left\{-\beta \tau^{Y}\right\} g_{4}\left(d_{4}\right) \mathbf{1}_{\left\{\tau_{1}^{Y}>\tau_{4}^{Y}\right\}} \mathbf{1}_{\left\{\tau_{4}^{Y}<t\right\}}\right) \\
&= \alpha_{1} \mathrm{E}\left(\exp \left\{-\beta \tau^{Y}\right\} \mathbf{1}_{\left\{\tau_{1}^{Y}<\tau_{4}^{Y}\right\}} \mathbf{1}_{\left\{\tau_{1}^{Y}<t\right\}}\right)+\alpha_{4} \mathrm{E}\left(\exp \left\{-\beta \tau^{Y}\right\} \mathbf{1}_{\left\{\tau_{1}^{Y}>\tau_{4}^{Y}\right\}} \mathbf{1}_{\left\{\tau_{4}^{Y}<t\right\}}\right)
\end{aligned}
$$

We also have

$$
\mathrm{E}\left(M_{t} \mathbf{1}_{\left\{\tau^{Y}>t\right\}}\right)=\mathrm{e}^{-\beta t} \mathrm{E}\left(g_{Z_{t}^{Y}}\left(V_{t}^{Y}\right) \mathbf{1}_{\left\{\tau^{Y}>t\right\}}\right),
$$

where $Z_{t}^{Y}$ can take the value $1,2,3$, or 4 . 
When $Z_{t}^{Y}=1$ or 4 , since $\tau^{Y}>t$, we have $0 \leq V_{t}^{Y}<d_{1} \wedge d_{4}$. According to the definition of $g_{i}(\mu)$ in (13), $g_{1}\left(V_{t}^{Y}\right)$ and $g_{4}\left(V_{t}^{Y}\right)$ are bounded. When $Z_{t}^{Y}=2$ or 3 , since $\lim _{d_{2} \rightarrow \infty} g_{2}\left(d_{2}\right)=$ $\lim _{d_{3} \rightarrow \infty} g_{3}\left(d_{3}\right)=0$, and looking at (13) with $d_{2}$ and $d_{3}$ replaced by $\infty, g_{2}\left(V_{t}^{Y}\right)$ and $g_{3}\left(V_{t}^{Y}\right)$ are bounded. Therefore,

$$
\lim _{t \rightarrow \infty} \mathrm{E}\left(M_{t} \mathbf{1}_{\left\{\tau^{Y}>t\right\}}\right)=0 .
$$

Hence, we have

$$
\lim _{t \rightarrow \infty} \mathrm{E}\left(M_{\tau^{Y} \wedge t}\right)=\alpha_{1} \mathrm{E}\left(\mathrm{e}^{-\beta \tau^{Y}} \mathbf{1}_{\left\{\tau_{1}^{Y}<\tau_{4}^{Y}\right\}}\right)+\alpha_{4} \mathrm{E}\left(\mathrm{e}^{-\beta \tau^{Y}} \mathbf{1}_{\left\{\tau_{1}^{Y}>\tau_{4}^{Y}\right\}}\right) .
$$

The left-hand side of (16) gives

$$
\lim _{t \rightarrow \infty} \mathrm{E}\left(M_{0}\right)=\mathrm{E}\left(M_{0}\right)= \begin{cases}g_{1}(0), & Y_{0}^{(\varepsilon)}=l_{1}+\varepsilon, \\ g_{4}(0), & Y_{0}^{(\varepsilon)}=l_{2}-\varepsilon .\end{cases}
$$

By taking $\alpha_{1}=1, \alpha_{4}=0$ and $\alpha_{1}=0, \alpha_{4}=1$, then, when $Y_{0}^{(\varepsilon)}=l_{1}+\varepsilon$,

$$
\begin{aligned}
\mathrm{E}(\exp (- & \left.\left.\beta \tau^{Y}\right) \mathbf{1}_{\left\{\tau_{1}^{Y}<\tau_{4}^{Y}\right\}}\right) \\
= & {\left[\exp \left\{-\beta d_{1}\right\} \bar{P}_{12}\left(d_{1}\right)\left(1-\hat{P}_{34}(\beta) \tilde{P}_{43}(\beta)\right)\right] } \\
& \times\left[1-\hat{P}_{21}(\beta) \tilde{P}_{12}(\beta)-\hat{P}_{34}(\beta) \tilde{P}_{43}(\beta)+\hat{P}_{21}(\beta) \tilde{P}_{12}(\beta) \hat{P}_{34}(\beta) \tilde{P}_{43}(\beta)\right. \\
& \left.\quad-\hat{P}_{31}(\beta) \tilde{P}_{43}(\beta) \hat{P}_{24}(\beta) \tilde{P}_{12}(\beta)\right]^{-1}, \\
\mathrm{E}(\exp \{- & \left.\left.\beta \tau^{Y}\right\} \mathbf{1}_{\left\{\tau_{1}^{Y}>\tau_{4}^{Y}\right\}}\right) \\
= & {\left[\exp \left\{-\beta d_{4}\right\} \bar{P}_{43}\left(d_{4}\right) \hat{P}_{24}(\beta) \tilde{P}_{12}(\beta)\right] } \\
& \times\left[1-\hat{P}_{21}(\beta) \tilde{P}_{12}(\beta)-\hat{P}_{34}(\beta) \tilde{P}_{43}(\beta)+\hat{P}_{21}(\beta) \tilde{P}_{12}(\beta) \hat{P}_{34}(\beta) \tilde{P}_{43}(\beta)\right. \\
& \left.\quad-\hat{P}_{31}(\beta) \tilde{P}_{43}(\beta) \hat{P}_{24}(\beta) \tilde{P}_{12}(\beta)\right]^{-1},
\end{aligned}
$$

and, when $Y_{0}^{(\varepsilon)}=l_{2}-\varepsilon$,

$$
\begin{aligned}
\mathrm{E}(\exp \{- & \left.\left.\beta \tau^{Y}\right\} \mathbf{1}_{\left\{\tau_{1}^{Y}<\tau_{4}^{Y}\right\}}\right) \\
= & {\left[\exp \left\{-\beta d_{1}\right\} \bar{P}_{12}\left(d_{1}\right) \hat{P}_{31}(\beta) \tilde{P}_{43}(\beta)\right] } \\
& \times\left[1-\hat{P}_{21}(\beta) \tilde{P}_{12}(\beta)-\hat{P}_{34}(\beta) \tilde{P}_{43}(\beta)+\hat{P}_{21}(\beta) \tilde{P}_{12}(\beta) \hat{P}_{34}(\beta) \tilde{P}_{43}(\beta)\right. \\
& \left.\quad-\hat{P}_{31}(\beta) \tilde{P}_{43}(\beta) \hat{P}_{24}(\beta) \tilde{P}_{12}(\beta)\right]^{-1}, \\
\mathrm{E}(\exp \{- & \left.\left.\beta \tau^{Y}\right\} \mathbf{1}_{\left\{\tau_{1}^{Y}>\tau_{4}^{Y}\right\}}\right) \\
= & {\left[\exp \left\{-\beta d_{4}\right\} \bar{P}_{43}\left(d_{4}\right)\left(1-\hat{P}_{21}(\beta) \tilde{P}_{12}(\beta)\right)\right] } \\
& \times\left[1-\hat{P}_{21}(\beta) \tilde{P}_{12}(\beta)-\hat{P}_{34}(\beta) \tilde{P}_{43}(\beta)+\hat{P}_{21}(\beta) \tilde{P}_{12}(\beta) \hat{P}_{34}(\beta) \tilde{P}_{43}(\beta)\right. \\
& \left.\quad-\hat{P}_{31}(\beta) \tilde{P}_{43}(\beta) \hat{P}_{24}(\beta) \tilde{P}_{12}(\beta)\right]^{-1} .
\end{aligned}
$$

\section{Main results}

In Section 2 we stated that the main difficulty with the Brownian motion is that the probability that $W_{t}^{\mu}$ will return to the origin at arbitrarily small times is 1 , and there are infinitely many excursions outside but also inside the barriers. We therefore introduced the new processes $Y_{t}^{(\mathcal{E})}$ and $\left(Z_{t}^{Y}, V_{t}^{Y}\right)$ with transition densities for $Z_{t}^{Y}$ defined in (6)-(12). 
In order to simplify the expressions, we define

$$
\Psi(x):=2 \sqrt{\pi} x \mathcal{N}(\sqrt{2} x)-\sqrt{\pi} x+\mathrm{e}^{-x^{2}},
$$

where $\mathcal{N}(\cdot)$ is the cumulative distribution function for the standard normal distribution.

Theorem 1. For a Brownian motion $W_{t}^{\mu}$, with $\tau_{1}^{W^{\mu}}, \tau_{4}^{W^{\mu}}$, and $\tau^{W^{\mu}}$ defined as in (1), (4), and (5), and $S_{t}=W_{t}^{\mu}$, we have the following Laplace transforms. When $W_{0}^{\mu}=l_{1}$,

$$
\begin{aligned}
\mathrm{E}\left(\exp \left\{-\beta \tau^{W^{\mu}}\right\} \mathbf{1}_{\left\{\tau_{1}^{W \mu}<\tau_{4}^{W \mu}\right\}}\right) & =\frac{G_{1}\left(d_{1}, d_{4}, \mu\right)}{G\left(d_{1}, d_{4}, \mu\right)}, \\
\mathrm{E}\left(\exp \left\{-\beta \tau^{W^{\mu}}\right\} \mathbf{1}_{\left\{\tau_{1}^{W^{\mu}}>\tau_{4}^{W^{\mu}}\right\}}\right) & =\frac{G_{2}\left(d_{4}, d_{1},-\mu\right)}{G\left(d_{1}, d_{4}, \mu\right)}, \\
\mathrm{E}\left(\exp \left\{-\beta \tau^{W^{\mu}}\right\}\right) & =\frac{G_{1}\left(d_{1}, d_{4}, \mu\right)+G_{2}\left(d_{4}, d_{1},-\mu\right)}{G\left(d_{1}, d_{4}, \mu\right)} .
\end{aligned}
$$

When $W_{0}^{\mu}=l_{2}$,

$$
\begin{aligned}
\mathrm{E}\left(\exp \left\{-\beta \tau^{W^{\mu}}\right\} \mathbf{1}_{\left\{\tau_{1}^{W^{\mu}}<\tau_{4}^{W^{\mu}}\right\}}\right) & =\frac{G_{2}\left(d_{1}, d_{4}, \mu\right)}{G\left(d_{1}, d_{4}, \mu\right)}, \\
\mathrm{E}\left(\exp \left\{-\beta \tau^{W^{\mu}}\right\} \mathbf{1}_{\left\{\tau_{1}^{W^{\mu}}>\tau_{4}^{W^{\mu}}\right\}}\right) & =\frac{G_{1}\left(d_{4}, d_{1},-\mu\right)}{G\left(d_{1}, d_{4}, \mu\right)}, \\
\mathrm{E}\left(\exp \left\{-\beta \tau^{W^{\mu}}\right\}\right) & =\frac{G_{1}\left(d_{4}, d_{1},-\mu\right)+G_{2}\left(d_{1}, d_{4}, \mu\right)}{G\left(d_{1}, d_{4}, \mu\right)} .
\end{aligned}
$$

Here

$$
\begin{aligned}
& G_{1}(x, y, z)=\exp \left\{-2\left(l_{1}-l_{2}\right) \sqrt{2 \beta+z^{2}}-\beta x\right\}\left\{\sqrt{y} \Psi\left(|z| \sqrt{\frac{x}{2}}\right)+z \sqrt{\frac{\pi x y}{2}}\right\} \\
& +\frac{\left(1-\exp \left\{-2\left(l_{1}-l_{2}\right) \sqrt{2 \beta+z^{2}}\right\}\right) \mathrm{e}^{-\beta x}}{2 \sqrt{2 \beta+z^{2}}}\left\{\Psi\left(|z| \sqrt{\frac{x}{2}}\right)+z \sqrt{\frac{\pi x}{2}}\right\} \\
& \times\left\{\sqrt{\frac{2}{\pi}} \Psi\left(\sqrt{\frac{\left(2 \beta+z^{2}\right) y}{2}}\right)+\sqrt{\left(2 \beta+z^{2}\right) y}\right\}, \\
& G_{2}(x, y, z)=\exp \left\{-\left(l_{1}-l_{2}\right)\left(\sqrt{2 \beta+z^{2}}-z\right)-\beta x\right\}\left\{\sqrt{y} \Psi\left(|z| \sqrt{\frac{x}{2}}\right)+z \sqrt{\frac{\pi x y}{2}}\right\}, \\
& G(x, y, z)=\exp \left\{-2\left(l_{1}-l_{2}\right) \sqrt{2 \beta+z^{2}}\right\} \\
& \times\left\{\sqrt{y} \Psi\left(\sqrt{\frac{\left(2 \beta+z^{2}\right) x}{2}}\right)+\sqrt{x} \Psi\left(\sqrt{\frac{\left(2 \beta+z^{2}\right) y}{2}}\right)\right\} \\
& +\frac{\left(1-\exp \left\{-2\left(l_{1}-l_{2}\right) \sqrt{2 \beta+z^{2}}\right\}\right)}{2 \sqrt{2 \beta+z^{2}}} \\
& \times\left\{\Psi\left(\sqrt{\frac{\left(2 \beta+z^{2}\right) x}{2}}\right)+\sqrt{\frac{\left(2 \beta+z^{2}\right) \pi x}{2}}\right\} \\
& \times\left\{\sqrt{\frac{2}{\pi}} \Psi\left(\sqrt{\frac{\left(2 \beta+z^{2}\right) y}{2}}\right)+\sqrt{\left(2 \beta+z^{2}\right) y}\right\} .
\end{aligned}
$$


Proof. We apply the transition densities in (6)-(12) to the results in (17)-(20) and take the limit as $\varepsilon \rightarrow 0$. According to the definition of $Y^{(\varepsilon)}$, we know that

$$
Y_{t}^{(\varepsilon)} \rightarrow W_{t}^{\mu} \quad \text { almost surely for all } t .
$$

As we saw in [6], since $Y_{t}^{(\varepsilon)} \rightarrow W_{t}^{\mu}$ almost surely for all $t$, by taking the limit $\varepsilon \rightarrow 0$, the quantities defined based on $Y_{t}^{(\varepsilon)}$ converge to those based on Brownian motion with drift. Therefore, we will obtain the results given in (21), (22), (24), and (25). We can thus obtain (23) and (26) by

$$
\mathrm{E}\left(\exp \left\{-\beta \tau^{W^{\mu}}\right\}\right)=\mathrm{E}\left(\exp \left\{-\beta \tau^{W^{\mu}}\right\} \mathbf{1}_{\left\{\tau_{1}^{W^{\mu}}<\tau_{4}^{W^{\mu}}\right\}}\right)+\mathrm{E}\left(\exp \left\{-\beta \tau^{W^{\mu}}\right\} \mathbf{1}_{\left\{\tau_{1}^{W^{\mu}}>\tau_{4}^{W^{\mu}}\right\}}\right) .
$$

Corollary 1. For a standard Brownian motion $(\mu=0)$, we have the following Laplace transforms. When $W_{0}=l_{1}$,

$$
\begin{aligned}
\mathrm{E}\left(\exp \left\{-\beta \tau^{W^{\mu}}\right\} \mathbf{1}_{\left\{\tau_{1}^{W}<\tau_{4}^{W}\right\}}\right) & =\frac{G_{1}\left(d_{1}, d_{4}, 0\right)}{G\left(d_{1}, d_{4}, 0\right)}, \\
\mathrm{E}\left(\exp \left\{-\beta \tau^{W^{\mu}}\right\} \mathbf{1}_{\left\{\tau_{1}^{W}>\tau_{4}^{W}\right\}}\right) & =\frac{G_{2}\left(d_{4}, d_{1}, 0\right)}{G\left(d_{1}, d_{4}, 0\right)} \\
\mathrm{E}\left(\exp \left\{-\beta \tau^{W^{\mu}}\right\}\right) & =\frac{G_{1}\left(d_{1}, d_{4}, 0\right)+G_{2}\left(d_{4}, d_{1}, 0\right)}{G\left(d_{1}, d_{4}, 0\right)} .
\end{aligned}
$$

When $W_{0}=l_{2}$,

$$
\begin{aligned}
\mathrm{E}\left(\exp \left\{-\beta \tau^{W^{\mu}}\right\} \mathbf{1}_{\left\{\tau_{1}^{W}<\tau_{4}^{W}\right\}}\right) & =\frac{G_{2}\left(d_{1}, d_{4}, 0\right)}{G\left(d_{1}, d_{4}, 0\right)}, \\
\mathrm{E}\left(\exp \left\{-\beta \tau^{W^{\mu}}\right\} \mathbf{1}_{\left\{\tau_{1}^{W}>\tau_{4}^{W}\right\}}\right) & =\frac{G_{1}\left(d_{4}, d_{1}, 0\right)}{G\left(d_{1}, d_{4}, 0\right)} \\
\mathrm{E}\left(\exp \left\{-\beta \tau^{W^{\mu}}\right\}\right) & =\frac{G_{1}\left(d_{4}, d_{1}, 0\right)+G_{2}\left(d_{1}, d_{4}, 0\right)}{G\left(d_{1}, d_{4}, 0\right)} .
\end{aligned}
$$

Here

$$
\begin{aligned}
G_{1}(x, y, 0)= & \exp \left\{-2\left(l_{1}-l_{2}\right) \sqrt{2 \beta}-\beta x\right\} \sqrt{y} \\
& +\frac{\left(1-\exp \left\{-2\left(l_{1}-l_{2}\right) \sqrt{2 \beta}\right\}\right) \mathrm{e}^{-\beta x}}{2 \sqrt{2 \beta}}\left\{\sqrt{\frac{2}{\pi}} \Psi(\sqrt{\beta y})+\sqrt{2 \beta y}\right\}, \\
G_{2}(x, y, 0)= & \exp \left\{-\left(l_{1}-l_{2}\right) \sqrt{2 \beta}-\beta x\right\} \sqrt{y}, \\
G(x, y, 0)= & \exp \left\{-2\left(l_{1}-l_{2}\right) \sqrt{2 \beta}\right\}\{\sqrt{y} \Psi(\sqrt{\beta x})+\sqrt{x} \Psi(\sqrt{\beta y})\} \\
+ & \frac{\left(1-\exp \left\{-2\left(l_{1}-l_{2}\right) \sqrt{2 \beta}\right\}\right)}{2 \sqrt{2 \beta}}\{\Psi(\sqrt{\beta x})+\sqrt{\beta \pi x}\} \\
& \times\left\{\sqrt{\frac{2}{\pi}} \Psi(\sqrt{\beta y})+\sqrt{2 \beta y}\right\} .
\end{aligned}
$$

Remark 1. By taking the limit $l_{1}-l_{2} \rightarrow 0$, we obtain the result for the single-barrier two-sided excursion case, as in [6].

Remark 2. If we only want to consider the excursion above a barrier, we can let $l_{2} \rightarrow-\infty$. Similarly, for the excursion below a barrier, we can let $l_{1} \rightarrow+\infty$. These results have been shown in [6]. 
Corollary 2. For a Brownian motion $W_{t}^{\mu}$, with $\tau^{W^{\mu}}$ defined as in (5) and $S_{t}=W_{t}^{\mu}$, we have the following Laplace transforms. When $W_{0}^{\mu}=x_{0}, x_{0}>l_{1}$,

$$
\begin{aligned}
& \mathrm{E}\left(\exp \left\{-\beta \tau^{W^{\mu}}\right\}\right) \\
& =\left\{\exp \left\{-\left(\mu+\sqrt{2 \beta+\mu^{2}}\right)\left(x_{0}-l_{1}\right)\right\} \mathcal{N}\left(\sqrt{\left(2 \beta+\mu^{2}\right) d_{1}}-\frac{x_{0}-l_{1}}{\sqrt{d_{1}}}\right)\right. \\
& \left.\quad+\exp \left\{-\left(\mu-\sqrt{2 \beta+\mu^{2}}\right)\left(x_{0}-l_{1}\right)\right\} \mathcal{N}\left(-\sqrt{\left(2 \beta+\mu^{2}\right) d_{1}}-\frac{x_{0}-l_{1}}{\sqrt{d_{1}}}\right)\right\} \\
& \quad \times \frac{G_{1}\left(d_{1}, d_{4}, \mu\right)+G_{2}\left(d_{4}, d_{1},-\mu\right)}{G\left(d_{1}, d_{4}, \mu\right)} \\
& +\mathrm{e}^{-\beta d_{1}}\left\{1-\exp \left\{-(\mu+|\mu|)\left(x_{0}-l_{1}\right)\right\} \mathcal{N}\left(|\mu| \sqrt{d_{1}}-\frac{x_{0}-l_{1}}{\sqrt{d_{1}}}\right)\right. \\
& \left.\quad-\exp \left\{-(\mu-|\mu|)\left(x_{0}-l_{1}\right)\right\} \mathcal{N}\left(-|\mu| \sqrt{d_{1}}-\frac{x_{0}-l_{1}}{\sqrt{d_{1}}}\right)\right\} .
\end{aligned}
$$

When $W_{0}^{\mu}=x_{0}, l_{2} \leq x_{0} \leq l_{1}$,

$$
\begin{aligned}
& \mathrm{E}\left(\exp \left\{-\beta \tau^{W^{\mu}}\right\}\right)
\end{aligned}
$$

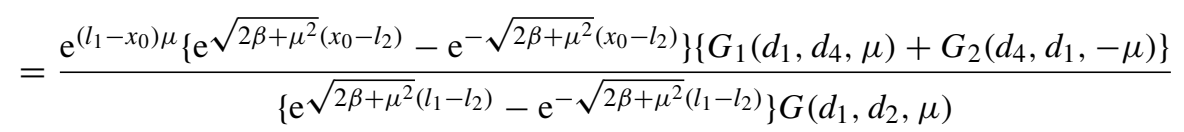

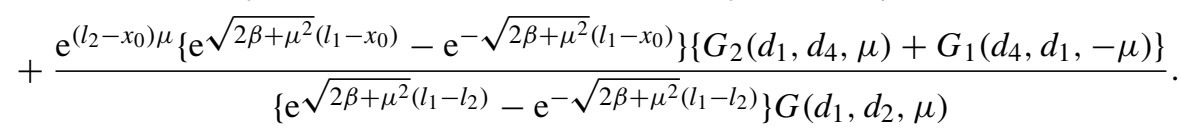

When $W_{0}^{\mu}=x_{0}, x_{0}<l_{2}$,

$$
\begin{aligned}
& \mathrm{E}\left(\exp \left(-\beta \tau^{W^{\mu}}\right)\right) \\
& =\left\{\exp \left\{\left(\mu-\sqrt{2 \beta+\mu^{2}}\right)\left(l_{2}-x\right)\right\} \mathcal{N}\left(\sqrt{\left(2 \beta+\mu^{2}\right) d_{4}}-\frac{l_{2}-x}{\sqrt{d_{4}}}\right)\right. \\
& \left.\quad+\exp \left\{\left(\mu+\sqrt{2 \beta+\mu^{2}}\right)\left(l_{2}-x\right)\right\} \mathcal{N}\left(-\sqrt{\left(2 \beta+\mu^{2}\right) d_{4}}-\frac{l_{2}-x}{\sqrt{d_{4}}}\right)\right\} \\
& \quad \times \frac{G_{1}\left(d_{4}, d_{1},-\mu\right)+G_{2}\left(d_{1}, d_{4}, \mu\right)}{G\left(d_{1}, d_{4}, \mu\right)} \\
& +\mathrm{e}^{-\beta d_{4}}\left\{1-\exp \left\{(\mu-|\mu|)\left(l_{2}-x\right)\right\} \mathcal{N}\left(|\mu| \sqrt{d_{4}}-\frac{l_{2}-x}{\sqrt{d_{4}}}\right)\right. \\
& \left.\quad-\exp \left\{(\mu+|\mu|)\left(l_{2}-x\right)\right\} \mathcal{N}\left(-|\mu| \sqrt{d_{4}}-\frac{l_{2}-x}{\sqrt{d_{4}}}\right)\right\} .
\end{aligned}
$$

Proof. We will first prove the case when $x_{0}>l_{1}$. Define $T=\inf \left\{t \mid W_{t}^{\mu}=l_{1}\right\}$, i.e. the first time $W_{t}^{\mu}$ hits $l_{1}$. By definition we have $\tau^{W^{\mu}}=d_{1}$ if $T \geq d_{1}$ and $\tau^{W^{\mu}}=T+\tau^{W^{\mu}}$ if $T<d_{1}$, where $\tilde{W}^{\mu}$ here stands for a Brownian motion with drift started from $l_{1}$. As a result,

$$
\begin{aligned}
\mathrm{E}\left(\exp \left\{-\beta \tau^{W^{\mu}}\right\}\right) & =\mathrm{E}\left(\exp \left\{-\beta \tau^{W^{\mu}}\right\} \mathbf{1}_{\left\{T \geq d_{1}\right\}}\right)+\mathrm{E}\left(\exp \left\{-\beta \tau^{W^{\mu}}\right\} \mathbf{1}_{\left\{T<d_{1}\right\}}\right) \\
& =\mathrm{e}^{-\beta d_{1}} \mathrm{P}\left(T \geq d_{1}\right)+\mathrm{E}\left(\mathrm{e}^{-\beta T} \mathbf{1}_{\left\{T<d_{1}\right\}}\right) \mathrm{E}\left(\exp \left\{-\beta \tau^{W^{\mu}}\right\}\right)
\end{aligned}
$$


The term $\mathrm{E}\left(\exp \left\{-\beta \tau^{\widetilde{W}^{\mu}}\right\}\right)$ has been calculated in Theorem 1 (see (23)). The density for $T$ was given in [2, Equation 2.0.2] as

$$
p_{x_{0}}=\frac{\left|l_{1}-x_{0}\right|}{\sqrt{2 \pi t^{3}}} \exp \left\{-\frac{\left(l_{i}-x_{0}-\mu t\right)^{2}}{2 t}\right\} .
$$

We can therefore calculate

$$
\begin{aligned}
\mathrm{P}\left(T \geq d_{1}\right)= & 1-\exp \left\{-(\mu+|\mu|)\left(x_{0}-l_{1}\right)\right\} \mathcal{N}\left(|\mu| \sqrt{d_{1}}-\frac{x_{0}-l_{1}}{\sqrt{d_{1}}}\right) \\
& -\exp \left\{-(\mu-|\mu|)\left(x_{0}-l_{1}\right)\right\} \mathcal{N}\left(-|\mu| \sqrt{d_{1}}-\frac{x_{0}-l_{1}}{\sqrt{d_{1}}}\right), \\
\mathrm{E}\left(\mathrm{e}^{-\beta T} \mathbf{1}_{\left\{T<d_{1}\right\}}\right)= & \exp \left\{-\left(\mu+\sqrt{2 \beta+\mu^{2}}\right)\left(x_{0}-l_{1}\right)\right\} \mathcal{N}\left(\sqrt{\left(2 \beta+\mu^{2}\right) d_{1}}-\frac{x_{0}-l_{1}}{\sqrt{d_{1}}}\right) \\
& +\exp \left\{-\left(\mu-\sqrt{2 \beta+\mu^{2}}\right)\left(x_{0}-l_{1}\right)\right\} \mathcal{N}\left(-\sqrt{\left(2 \beta+\mu^{2}\right) d_{1}}-\frac{x_{0}-l_{1}}{\sqrt{d_{1}}}\right) .
\end{aligned}
$$

We therefore obtain the result in (27). For the case when $x_{0}<l_{2}$, we can apply the same argument.

When $l_{2} \leq x_{0} \leq l_{1}$, we define $\widetilde{T}=\inf \left(t \mid W_{t}^{\mu} \notin\left(l_{2}, l_{1}\right)\right)$. By definition we have $\tau^{W^{\mu}}=$ $T+\tau^{\widetilde{W}^{\mu}}$ if $W_{T}^{\mu}=l_{1}$ and $\tau^{W^{\mu}}=T+\tau{\underline{W^{\mu}}}^{\mu}$ if $W_{T}^{\mu}=l_{2}$, where $\underline{W}^{\mu}$ stands for a Brownian motion with drift started from $l_{2}$. Consequently,

$$
\begin{aligned}
& \mathrm{E}\left(\operatorname { e x p } \left\{-\beta \tau^{\left.\left.W^{\mu}\right\}\right)}\right.\right. \\
& \quad=\mathrm{E}\left(\mathrm{e}^{-\beta T} \exp \left\{-\beta \tau^{\tilde{W}^{\mu}}\right\} \mathbf{1}_{\left\{T=l_{1}\right\}}\right)+\mathrm{E}\left(\mathrm{e}^{-\beta T} \exp \left\{-\beta \tau^{\underline{W}^{\mu}}\right\} \mathbf{1}_{\left\{T=l_{2}\right\}}\right) \\
& =\mathrm{E}\left(\mathrm{e}^{-\beta T} \mathbf{1}_{\left\{T=l_{1}\right\}}\right) \mathrm{E}\left(\exp \left\{-\beta \tau^{\left.\widetilde{W}^{\mu}\right\}}\right)+\mathrm{E}\left(\mathrm{e}^{-\beta T} \mathbf{1}_{\left\{T=l_{2}\right\}}\right) \mathrm{E}\left(\exp \left\{-\beta \tau^{\underline{W}^{\mu}}\right\}\right) .\right.
\end{aligned}
$$

The terms $\mathrm{E}\left(\exp \left\{-\beta \tau^{\tilde{W}^{\mu}}\right\}\right)$ and $\mathrm{E}\left(\exp \left\{-\beta \tau \underline{\underline{W}}^{\mu}\right\}\right)$ have been obtained in Theorem 1 (see (23) and (26)). According to [2, Equation 3.0.5], we have

$$
\begin{aligned}
& \mathrm{E}\left(\mathrm{e}^{-\beta T} \mathbf{1}_{\left\{T=l_{1}\right\}}\right)=\frac{\mathrm{e}^{\left(l_{1}-x_{0}\right) \mu}\left\{\mathrm{e}^{\sqrt{2 \beta+\mu^{2}}\left(x_{0}-l_{2}\right)}-\mathrm{e}^{-\sqrt{2 \beta+\mu^{2}}\left(x_{0}-l_{2}\right)}\right\}}{\mathrm{e}^{\sqrt{2 \beta+\mu^{2}}\left(l_{1}-l_{2}\right)}-\mathrm{e}^{-\sqrt{2 \beta+\mu^{2}}\left(l_{1}-l_{2}\right)}}, \\
& \mathrm{E}\left(\mathrm{e}^{-\beta T} \mathbf{1}_{\left\{T=l_{2}\right\}}\right)=\frac{\mathrm{e}^{\left(l_{2}-x_{0}\right) \mu}\left\{\mathrm{e}^{\sqrt{2 \beta+\mu^{2}}\left(l_{1}-x_{0}\right)}-\mathrm{e}^{-\sqrt{2 \beta+\mu^{2}}\left(l_{1}-x_{0}\right)}\right\}}{\mathrm{e}^{\sqrt{2 \beta+\mu^{2}}\left(l_{1}-l_{2}\right)}-\mathrm{e}^{-\sqrt{2 \beta+\mu^{2}}\left(l_{1}-l_{2}\right)}} .
\end{aligned}
$$

We have therefore obtained (28).

Theorem 2. The probability that $W_{t}^{\mu}$ with $W_{0}^{\mu}=x_{0}, l_{2} \leq x_{0} \leq l_{1}$, achieves an excursion above $l_{1}$ with length at least $d_{1}$ before it achieves an excursion below $l_{2}$ with length at least $d_{4}$ is

$$
\begin{aligned}
\mathrm{P}\left(\tau_{1}^{W^{\mu}}<\tau_{4}^{W^{\mu}}\right)= & \frac{\mathrm{e}^{\left(l_{1}-x_{0}\right) \mu}\left\{\mathrm{e}^{|\mu|\left(x_{0}-l_{2}\right)}-\mathrm{e}^{-|\mu|\left(x_{0}-l_{2}\right)}\right\} F_{1}\left(d_{1}, d_{4}, \mu\right)}{\left\{\mathrm{e}^{|\mu|\left(l_{1}-l_{2}\right)}-\mathrm{e}^{-|\mu|\left(l_{1}-l_{2}\right)}\right\} F\left(d_{1}, d_{4}, \mu\right)} \\
& +\frac{\mathrm{e}^{\left(l_{2}-x_{0}\right) \mu}\left\{\mathrm{e}^{|\mu|\left(l_{1}-x_{0}\right)}-\mathrm{e}^{-|\mu|\left(l_{1}-x_{0}\right)}\right\} F_{2}\left(d_{1}, d_{4}, \mu\right)}{\left\{\mathrm{e}^{|\mu|\left(l_{1}-l_{2}\right)}-\mathrm{e}^{-|\mu|\left(l_{1}-l_{2}\right)}\right\} F\left(d_{1}, d_{4}, \mu\right)}, \\
\mathrm{P}\left(\tau_{1}^{W^{\mu}}>\tau_{4}^{W^{\mu}}\right)= & \frac{\mathrm{e}^{\left(l_{1}-x_{0}\right) \mu}\left\{\mathrm{e}^{|\mu|\left(x_{0}-l_{2}\right)}-\mathrm{e}^{-|\mu|\left(x_{0}-l_{2}\right)}\right\} F_{2}\left(d_{4}, d_{1},-\mu\right)}{\left\{\mathrm{e}^{|\mu|\left(l_{1}-l_{2}\right)}-\mathrm{e}^{-|\mu|\left(l_{1}-l_{2}\right)}\right\} F\left(d_{1}, d_{4}, \mu\right)} \\
& +\frac{\mathrm{e}^{\left(l_{2}-x_{0}\right) \mu}\left\{\mathrm{e}^{|\mu|\left(l_{1}-x_{0}\right)}-\mathrm{e}^{-|\mu|\left(l_{1}-x_{0}\right)}\right\} F_{1}\left(d_{4}, d_{1},-\mu\right)}{\left\{\mathrm{e}^{|\mu|\left(l_{1}-l_{2}\right)}-\mathrm{e}^{-|\mu|\left(l_{1}-l_{2}\right)}\right\} F\left(d_{1}, d_{4}, \mu\right)},
\end{aligned}
$$


where

$$
\begin{aligned}
F_{1}(x, y, z)= & \exp \left\{-2\left(l_{1}-l_{2}\right)|z|\right\}\left\{\sqrt{y} \Psi\left(|z| \sqrt{\frac{x}{2}}\right)+z \sqrt{\frac{\pi x y}{2}}\right\} \\
+ & \left.\frac{\left(1-\exp \left\{-2\left(l_{1}-l_{2}\right)|z|\right)\right.}{2|z|}\right\}\left\{\left(|z| \sqrt{\frac{x}{2}}\right)+z \sqrt{\frac{\pi x}{2}}\right\} \\
& \times\left\{\sqrt{\frac{2}{\pi}} \Psi\left(|z| \sqrt{\frac{y}{2}}\right)+|z| \sqrt{y}\right\}, \\
F_{2}(x, y, z)= & \exp \left\{-\left(l_{1}-l_{2}\right)(|z|-z)\right\}\left\{\sqrt{y} \Psi\left(|z| \sqrt{\frac{x}{2}}\right)+z \sqrt{\frac{\pi x y}{2}}\right\}, \\
F(x, y, z)= & \exp \left\{-2\left(l_{1}-l_{2}\right)|z|\right\}\left\{\sqrt{y} \Psi\left(|z| \sqrt{\frac{x}{2}}\right)+\sqrt{x} \Psi\left(|z| \sqrt{\frac{y}{2}}\right)\right\} \\
+ & \left.\frac{\left(1-\exp \left\{-2\left(l_{1}-l_{2}\right)|z|\right)\right.}{2|z|}\right\}\left\{\Psi\left(|z| \sqrt{\frac{x}{2}}\right)+|z| \sqrt{\frac{\pi x}{2}}\right\} \\
& \times\left\{\sqrt{\frac{2}{\pi}} \Psi\left(|z| \sqrt{\frac{y}{2}}\right)+|z| \sqrt{y}\right\} .
\end{aligned}
$$

Proof. From Theorem 1 and (28), we actually know that, when $W_{0}^{\mu}=x_{0}, l_{2} \leq x_{0} \leq l_{1}$,

$$
\begin{aligned}
\mathrm{E}\left(\exp \left\{-\beta \tau^{W^{\mu}}\right\} \mathbf{1}_{\left\{\tau_{1}^{W^{\mu}}<\tau_{4}^{W^{\mu}}\right\}}\right)= & \frac{\mathrm{e}^{\left(l_{1}-x_{0}\right) \mu}\left\{\mathrm{e}^{|\mu|\left(x_{0}-l_{2}\right)}-\mathrm{e}^{-|\mu|\left(x_{0}-l_{2}\right)}\right\} G_{1}\left(d_{1}, d_{4}, \mu\right)}{\left\{\mathrm{e}^{|\mu|\left(l_{1}-l_{2}\right)}-\mathrm{e}^{-|\mu|\left(l_{1}-l_{2}\right)}\right\} G\left(d_{1}, d_{4}, \mu\right)} \\
& +\frac{\mathrm{e}^{\left(l_{2}-x_{0}\right) \mu}\left\{\mathrm{e}^{|\mu|\left(l_{1}-x_{0}\right)}-\mathrm{e}^{-|\mu|\left(l_{1}-x_{0}\right)}\right\} G_{2}\left(d_{1}, d_{4}, \mu\right)}{\left\{\mathrm{e}^{|\mu|\left(l_{1}-l_{2}\right)}-\mathrm{e}^{-|\mu|\left(l_{1}-l_{2}\right)}\right\} G\left(d_{1}, d_{4}, \mu\right)}, \\
\mathrm{E}\left(\exp \left\{-\beta \tau^{W^{\mu}}\right\} \mathbf{1}_{\left\{\tau_{1}^{W \mu}>\tau_{4}^{W^{\mu}}\right\}}\right)= & \frac{\mathrm{e}^{\left(l_{1}-x_{0}\right) \mu}\left\{\mathrm{e}^{|\mu|\left(x_{0}-l_{2}\right)}-\mathrm{e}^{-|\mu|\left(x_{0}-l_{2}\right)}\right\} G_{2}\left(d_{4}, d_{1},-\mu\right)}{\left\{\mathrm{e}^{|\mu|\left(l_{1}-l_{2}\right)}-\mathrm{e}^{-|\mu|\left(l_{1}-l_{2}\right)}\right\} G\left(d_{1}, d_{4}, \mu\right)} \\
& +\frac{\mathrm{e}^{\left(l_{2}-x_{0}\right) \mu}\left\{\mathrm{e}^{|\mu|\left(l_{1}-x_{0}\right)}-\mathrm{e}^{-|\mu|\left(l_{1}-x_{0}\right)}\right\} G_{1}\left(d_{4}, d_{1},-\mu\right)}{\left\{\mathrm{e}^{|\mu|\left(l_{1}-l_{2}\right)}-\mathrm{e}^{-|\mu|\left(l_{1}-l_{2}\right)}\right\} G\left(d_{1}, d_{4}, \mu\right)} .
\end{aligned}
$$

Setting $\beta=0$ in (29) and (30) yields the results.

Theorem 2 leads to the following remarkable result.

Corollary 3. For a standard Brownian motion $W_{t}$ with $W_{0}=x_{0}, l_{2} \leq x_{0} \leq l_{1}$, we have

$$
\begin{aligned}
& \mathrm{P}\left(\tau_{1}^{W}<\tau_{4}^{W}\right)=\frac{\sqrt{d_{4}}+\left(x_{0}-l_{2}\right) \sqrt{2 / \pi}}{\sqrt{d_{1}}+\sqrt{d_{4}}+\left(l_{1}-l_{2}\right) \sqrt{2 / \pi}}, \\
& \mathrm{P}\left(\tau_{1}^{W}>\tau_{4}^{W}\right)=\frac{\sqrt{d_{1}}+\left(l_{1}-x_{0}\right) \sqrt{2 / \pi}}{\sqrt{d_{1}}+\sqrt{d_{4}}+\left(l_{1}-l_{2}\right) \sqrt{2 / \pi}} .
\end{aligned}
$$

Remark 3. When we take $l_{1} \rightarrow 0, l_{2} \rightarrow 0$, and $x_{0} \rightarrow 0$, we can obtain the results for the one-barrier case, as in [6].

Remark 4. We observe that the formulae in Corollary 3 are linear in the starting point $x_{0}$, as is also the case for the exit probabilities of a standard Brownian motion or, more generally, a diffusion in its natural state (see [3, Section 16.5]). If we set $d_{1} \rightarrow 0$ and $d_{4} \rightarrow 0$, we recover $\left(x_{0}-l_{2}\right) /\left(l_{1}-l_{2}\right)$ and $\left(l_{1}-x_{0}\right) /\left(l_{1}-l_{2}\right)$, the exit probabilities.

We will now extend Corollary 2 to obtain the joint distribution of $W_{t}$ and $\tau^{W}$ at an exponential time. This is an application of (28) and Girsanov's theorem. 
Theorem 3. For a standard Brownian motion $W_{t}$ with $W_{0}=x_{0}, l_{2} \leq x_{0} \leq l_{1}$, and $\tau^{W}$ defined as in (3) with $S_{t}=W_{t}$, we have the following results. For the case in which $x>l_{1}$,

$$
\begin{aligned}
\mathrm{P}\left(W_{\tilde{T}} \in \mathrm{d} x, \tau^{W}<\tilde{T}\right)= & \left(a_{1}\left(x_{0}\right) f\left(x-l_{1}, d_{1}\right)+a_{2}\left(x_{0}\right) f\left(x-l_{2}, d_{4}\right)\right. \\
& \left.+a_{1}\left(x_{0}\right) h\left(x-l_{1}, d_{1}\right)\right) \mathrm{d} x .
\end{aligned}
$$

For the case in which $l_{2} \leq x \leq l_{1}$,

$$
\mathrm{P}\left(W_{\tilde{T}} \in \mathrm{d} x, \tau^{W}<\tilde{T}\right)=\left(a_{1}\left(x_{0}\right) f\left(x-l_{1}, d_{1}\right)+a_{2}\left(x_{0}\right) f\left(x-l_{2}, d_{4}\right)\right) \mathrm{d} x .
$$

For the case in which $x<l_{2}$,

$$
\begin{aligned}
\mathrm{P}\left(W_{\tilde{T}} \in \mathrm{d} x, \tau^{W}<\tilde{T}\right)= & \left(a_{1}\left(x_{0}\right) f\left(x-l_{1}, d_{1}\right)+a_{2}\left(x_{0}\right) f\left(x-l_{2}, d_{4}\right)\right. \\
& \left.+a_{2}\left(x_{0}\right) h\left(x-l_{2}, d_{4}\right)\right) \mathrm{d} x .
\end{aligned}
$$

Here $\tilde{T}$ is a random variable with an exponential distribution of parameter $\gamma$ that is independent of $W_{t}$ and

$$
\begin{aligned}
f(x, y)= & \frac{\mathrm{e}^{-\sqrt{2 \gamma}|x|}}{\sqrt{2 \gamma}}-\mathrm{e}^{\gamma y-\sqrt{2 \gamma}|x|} \sqrt{2 \pi y} \mathcal{N}(-\sqrt{2 \gamma y}) \\
h(x, y)= & \sqrt{2 \pi y} \mathrm{e}^{\gamma y}\left\{\mathrm{e}^{-\sqrt{2 \gamma}|x|} \mathcal{N}\left(\frac{|x|}{\sqrt{y}}-\sqrt{2 \gamma y}\right)-\mathrm{e}^{\sqrt{2 \gamma}|x|} \mathcal{N}\left(-\frac{|x|}{\sqrt{y}}-\sqrt{2 \gamma y}\right)\right\}, \\
a_{1}\left(x_{0}\right)= & \frac{\gamma\left\{\mathrm{e}^{\sqrt{2 \gamma}\left(x_{0}-l_{2}\right)}-\mathrm{e}^{-\sqrt{2 \gamma}\left(x_{0}-l_{2}\right)}\right\} b_{1}\left(d_{1}, d_{4}\right)}{G\left\{\mathrm{e}^{\sqrt{2 \gamma}\left(l_{1}-l_{2}\right)}-\mathrm{e}^{-\sqrt{2 \gamma}\left(l_{1}-l_{2}\right)}\right\}} \\
& +\frac{\gamma\left\{\mathrm{e}^{\sqrt{2 \gamma}\left(l_{1}-x_{0}\right)}-\mathrm{e}^{-\sqrt{2 \gamma}\left(l_{1}-x_{0}\right)}\right\} b_{2}\left(d_{1}, d_{4}\right)}{G\left\{\mathrm{e}^{\sqrt{2 \gamma}\left(l_{1}-l_{2}\right)}-\mathrm{e}^{-\sqrt{2 \gamma}\left(l_{1}-l_{2}\right)}\right\}}, \\
a_{2}\left(x_{0}\right)= & \frac{\gamma\left\{\mathrm{e}^{\sqrt{2 \gamma}\left(x_{0}-l_{2}\right)}-\mathrm{e}^{-\sqrt{2 \gamma}\left(x_{0}-l_{2}\right)}\right\} b_{2}\left(d_{4}, d_{1}\right)}{G\left\{\mathrm{e}^{\sqrt{2 \gamma}\left(l_{1}-l_{2}\right)}-\mathrm{e}^{-\sqrt{2 \gamma}\left(l_{1}-l_{2}\right)}\right\}} \\
& +\frac{\gamma\left\{\mathrm{e}^{\sqrt{2 \gamma}\left(l_{1}-x_{0}\right)}-\mathrm{e}^{-\sqrt{2 \gamma}\left(l_{1}-x_{0}\right)}\right\} b_{1}\left(d_{4}, d_{1}\right)}{G\left\{\mathrm{e}^{\sqrt{2 \gamma}\left(l_{1}-l_{2}\right)}-\mathrm{e}^{-\sqrt{2 \gamma}\left(l_{1}-l_{2}\right)}\right\}}, \\
b_{1}(x, y)= & \mathrm{e}^{-2\left(l_{1}-l_{2}\right) \sqrt{2 \gamma}-\gamma x} \sqrt{y}+\frac{1-\mathrm{e}^{-2 \gamma \sqrt{2 \gamma}}}{2 \sqrt{2 \gamma}} \mathrm{e}^{-\gamma x}\left\{\sqrt{\frac{2}{\pi}} \Psi(\sqrt{\gamma y})+\sqrt{2 \gamma y}\right\}, \\
b_{2}(x, y)= & \mathrm{e}^{-\left(l_{1}-l_{2}\right) \sqrt{2 \gamma}-\gamma x} \sqrt{y}, \\
G= & \mathrm{e}^{-2\left(l_{1}-l_{2}\right) \sqrt{2 \gamma}}\left\{\sqrt{d_{4}} \Psi\left(\sqrt{\gamma d_{1}}\right)+\sqrt{d_{1}} \Psi\left(\sqrt{\gamma d_{4}}\right)\right\} \\
& +\frac{\left(1-\mathrm{e}^{-2\left(l_{1}-l_{2}\right) \sqrt{2 \gamma}}\right)}{2 \sqrt{2 \gamma}}\left\{\Psi\left(\sqrt{\gamma d_{1}}\right)+\sqrt{\gamma \pi d_{1}}\right\}\left\{\sqrt{\left.\frac{2}{\pi} \Psi\left(\sqrt{\gamma d_{4}}\right)+\sqrt{2 \gamma d_{4}}\right\} .}\right.
\end{aligned}
$$

Proof. See Appendix A.

\section{Pricing double-barrier Parisian options}

We want to price a double-barrier Parisian call option with the current price of its underlying asset being $x, L_{1}<x<L_{2}$, the owner of which will obtain the right to exercise it when either 
the length of the excursion above the barrier $L_{1}$ reaches $d_{1}$, or the length of the excursion below the barrier $L_{2}$ reaches $d_{2}$ before $T$. Its price formula is given by

$$
\mathrm{DP}_{\text {min-in call }}=\mathrm{e}^{-r T} \mathrm{E}_{Q}\left(\left(S_{T}-K\right)^{+} \mathbf{1}_{\left\{\tau^{S}<T\right\}}\right),
$$

where $S$ is the underlying stock price, $Q$ denotes the risk neutral measure, and $\tau^{S}$ is defined with respect to barriers $L_{1}$ and $L_{2}$. The subscript min-in call means it is a call option which will be triggered when the minimum of two stopping times, $\tau_{1}^{S}$ and $\tau_{4}^{S}$, is less than $T$, i.e. $\tau^{S}<T$. We assume that $S$ is a geometric Brownian motion, i.e.

$$
\mathrm{d} S_{t}=r S_{t} \mathrm{~d} t+\sigma S_{t} \mathrm{~d} W_{t}, \quad S_{0}=x,
$$

where $L_{1}<x<L_{2}, r$ is the risk free rate, and $W_{t}$ with $W_{0}=0$ is a standard Brownian motion under $Q$. Set

$$
\begin{gathered}
m=\frac{1}{\sigma}\left(r-\frac{1}{2} \sigma^{2}\right), \quad b=\frac{1}{\sigma} \ln \left(\frac{K}{x}\right), \quad B_{t}=m t+W_{t}, \\
l_{1}=\frac{1}{\sigma} \ln \left(\frac{L_{1}}{x}\right), \quad l_{2}=\frac{1}{\sigma} \ln \left(\frac{L_{2}}{x}\right) .
\end{gathered}
$$

We have

$$
S_{t}=x \exp \left\{\left(r-\frac{1}{2} \sigma^{2}\right) t+\sigma W_{t}\right\}=x \exp \left\{\sigma\left(m t+W_{t}\right)\right\}=x \mathrm{e}^{\sigma B_{t}} .
$$

By applying Girsanov's theorem we have

$$
\mathrm{DP}_{\text {min-in call }}=\mathrm{e}^{-\left(r+m^{2} / 2\right) T} \mathrm{E}_{P}\left[\left(x \mathrm{e}^{\sigma B_{T}}-K\right)^{+} \mathrm{e}^{m B_{T}} \mathbf{1}_{\left\{\tau^{B}<T\right\}}\right],
$$

where $P$ is a new measure, under which $B_{t}$ is a standard Brownian motion with $B_{0}=0$, and $\tau^{B}$ is the stopping time defined with respect to barriers $l_{1}$ and $l_{2}$. We also define

$$
\mathrm{DP}_{\text {min-in call }}^{*}=\mathrm{e}^{\left(r+m^{2} / 2\right) T} \mathrm{DP}_{\text {min-in call }} \text {. }
$$

We are going to show that we can obtain the Laplace transform of $\mathrm{DP}_{\text {min-in call }}^{*}$ with respect to $T$, denoted by $\mathcal{L}_{T}$.

Firstly, assuming that $\tilde{T}$ is a random variable with an exponential distribution of parameter $\gamma$ which is independent of $W_{t}$, we have

$$
\begin{aligned}
& \mathrm{E}_{P}\left[\left(x \mathrm{e}^{\sigma B_{\tilde{T}}}-K\right)^{+} \mathrm{e}^{m B_{\tilde{T}}} \mathbf{1}_{\left\{\tau^{B}<\tilde{T}\right\}}\right] \\
& =\int_{b}^{\infty}\left(x \mathrm{e}^{\sigma y}-K\right) \mathrm{e}^{m y} \mathrm{P}\left(B_{\tilde{T}} \in \mathrm{d} y, \tau^{B}<\tilde{T}\right) \\
& =\int_{0}^{\infty} \gamma \mathrm{e}^{-\gamma T} \int_{b}^{\infty}\left(x \mathrm{e}^{\sigma y}-K\right) \mathrm{e}^{m y} \mathrm{P}\left(B_{T} \in \mathrm{d} y, \tau^{B}<T\right) \mathrm{d} T \\
& =\gamma \int_{0}^{\infty} \mathrm{e}^{-\gamma T} \mathrm{E}_{P}\left[\left(x \mathrm{e}^{\sigma B_{T}}-K\right)^{+} \mathrm{e}^{m B_{T}} \mathbf{1}_{\left\{\tau^{B}<T\right\}}\right] \mathrm{d} T \\
& =\gamma \mathcal{L}_{T} \text {. }
\end{aligned}
$$

Hence, we have

$$
\mathcal{L}_{T}=\frac{1}{\gamma} \int_{b}^{\infty}\left(x \mathrm{e}^{\sigma y}-K\right) \mathrm{e}^{m y} \mathrm{P}\left(B_{\tilde{T}} \in \mathrm{d} y, \tau^{B}<\tilde{T}\right) .
$$

Using the results of Theorem 3, this Laplace transform can be calculated explicitly. 
When $b \geq l_{1}$, i.e. $K \geq L_{1}$, we have

$$
\mathcal{L}_{T}=\frac{x}{\gamma} F_{1}(\sigma+m)-\frac{K}{\gamma} F_{1}(m),
$$

where

$$
\begin{aligned}
F_{1}(x)= & a_{1}(0)\left\{\frac{1}{\sqrt{2 \gamma}}-\mathrm{e}^{\gamma d_{1}} \sqrt{2 \pi d_{1}} \mathcal{N}\left(-\sqrt{2 \gamma d_{1}}\right)\right\} \frac{\exp \left\{\sqrt{2 \gamma} l_{1}+(x-\sqrt{2 \gamma}) b\right\}}{\sqrt{2 \gamma}-x} \\
+ & a_{2}(0)\left\{\frac{1}{\sqrt{2 \gamma}}-\mathrm{e}^{\gamma d_{4}} \sqrt{2 \pi d_{4}} \mathcal{N}\left(-\sqrt{2 \gamma d_{4}}\right)\right\} \frac{\exp \left\{\sqrt{2 \gamma} l_{2}+(x-\sqrt{2 \gamma}) b\right\}}{\sqrt{2 \gamma}-x} \\
+ & a_{1}(0) \sqrt{2 \pi d_{1}} \mathrm{e}^{\gamma d_{1}} \\
& \times\left\{\frac{2 x \exp \left\{x l_{1}-r d_{1}+d_{1} x^{2} / 2\right\} \mathcal{N}\left(x \sqrt{d_{1}}-\left(b-l_{1}\right) / \sqrt{d_{1}}\right)}{2 \gamma-x^{2}}\right. \\
& +\frac{\exp \left\{\sqrt{2 \gamma} l_{1}+(x-\sqrt{2 \gamma}) b\right\} \mathcal{N}\left(\left(b-l_{1}\right) / \sqrt{d_{1}}-\sqrt{2 \gamma d_{1}}\right)}{\sqrt{2 \gamma}-x} \\
& \left.+\frac{\exp \left\{-\sqrt{2 \gamma} l_{1}+(x+\sqrt{2 \gamma}) b\right\} \mathcal{N}\left(-\left(b-l_{1}\right) / \sqrt{d_{1}}-\sqrt{2 \gamma d_{1}}\right)}{\sqrt{2 \gamma}+x}\right\}
\end{aligned}
$$

When $l_{2}<b<l_{1}$, i.e. $L_{2}<K<L_{1}$, we have

$$
\mathcal{L}_{T}=\frac{x}{\gamma} F_{2}(\sigma+m)-\frac{K}{\gamma} F_{2}(m),
$$

where

$$
\begin{aligned}
F_{2}(x)= & \frac{2 a_{1}(0) \mathrm{e}^{l_{1} x}}{2 \gamma-x^{2}}\left\{1+x \sqrt{2 \pi d_{1}} \mathrm{e}^{d_{1} x^{2} / 2} \mathcal{N}\left(x \sqrt{d_{1}}\right)\right\} \\
& -a_{1}(0)\left\{\frac{1}{\sqrt{2 \gamma}}-\mathrm{e}^{\gamma d_{1}} \sqrt{2 \pi d_{1}} \mathcal{N}\left(-\sqrt{2 \gamma d_{1}}\right)\right\} \frac{\exp \left\{-\sqrt{2 \gamma} l_{1}+(x+\sqrt{2 \gamma}) b\right\}}{\sqrt{2 \gamma}-x} \\
& +a_{2}(0)\left\{\frac{1}{\sqrt{2 \gamma}}-\mathrm{e}^{\gamma d_{4}} \sqrt{2 \pi d_{4}} \mathcal{N}\left(-\sqrt{2 \gamma d_{4}}\right)\right\} \frac{\exp \left\{\sqrt{2 \gamma} l_{2}+(x-\sqrt{2 \gamma}) b\right\}}{\sqrt{2 \gamma}-x} .
\end{aligned}
$$

When $b \leq l_{2}$, i.e. $K \leq L_{2}$, we have

$$
\mathcal{L}_{T}=\frac{x}{\gamma} F_{3}(\sigma+m)-\frac{K}{\gamma} F_{3}(m),
$$

where

$$
\begin{aligned}
F_{2}(x)= & \frac{2 a_{1}(0) \mathrm{e}^{l_{1} x}}{2 \gamma-x^{2}}\left\{1+x \sqrt{2 \pi d_{1}} \mathrm{e}^{d_{1} x^{2} / 2} \mathcal{N}\left(x \sqrt{d_{1}}\right)\right\} \\
& -a_{1}(0)\left\{\frac{1}{\sqrt{2 \gamma}}-\mathrm{e}^{\gamma d_{1}} \sqrt{2 \pi d_{1}} \mathcal{N}\left(-\sqrt{2 \gamma d_{1}}\right)\right\} \frac{\exp \left\{-\sqrt{2 \gamma} l_{1}+(x+\sqrt{2 \gamma}) b\right\}}{\sqrt{2 \gamma}-x} \\
& +\frac{2 a_{2}(0) \mathrm{e}^{l_{2} x}}{2 \gamma-x^{2}}\left\{1-2 \sqrt{\pi d_{4} \gamma} \mathrm{e}^{d_{4} x^{2} / 2} \mathcal{N}\left(x \sqrt{d_{4}}\right)\right\} \\
& -a_{2}(0)\left\{\frac{1}{\sqrt{2 \gamma}}-\mathrm{e}^{\gamma d_{4}} \sqrt{2 \pi d_{4}} \mathcal{N}\left(-\sqrt{2 \gamma d_{4}}\right)\right\} \frac{\exp \left\{-\sqrt{2 \gamma} l_{2}+(x+\sqrt{2 \gamma}) b\right\}}{\sqrt{2 \gamma}-x}
\end{aligned}
$$




$$
\begin{aligned}
& +a_{2}(0) \sqrt{2 \pi d_{4}} \mathrm{e}^{\gamma d_{4}} \\
& +\times\left\{\frac{2 \sqrt{2 \gamma} \exp \left\{x l_{2}-r d_{4}+d_{4} x^{2} / 2\right\} \mathcal{N}\left(x \sqrt{d_{4}}-\left(b-l_{2}\right) / \sqrt{d_{4}}\right)}{2 \gamma-x^{2}}\right. \\
& \quad-\frac{\exp \left\{\sqrt{2 \gamma} l_{2}+(x-\sqrt{2 \gamma}) b\right\} \mathcal{N}\left(\left(b-l_{2}\right) / \sqrt{d_{4}}-\sqrt{2 \gamma d_{4}}\right)}{\sqrt{2 \gamma}-x} \\
& \left.\quad-\frac{\exp \left\{-\sqrt{2 \gamma} l_{2}+(x+\sqrt{2 \gamma}) b\right\} \mathcal{N}\left(-\left(b-l_{2}\right) / \sqrt{d_{4}}-\sqrt{2 \gamma d_{4}}\right)}{\sqrt{2 \gamma}+x}\right\} .
\end{aligned}
$$

Remark. The price can be calculated by numerical inversion of the Laplace transform.

So far, we have shown how to obtain the Laplace transform of

$$
\mathrm{DP}_{\text {min-call in }}^{*}=\mathrm{e}^{\left(r+m^{2} / 2\right) T} \mathrm{DP}_{\text {min-call in }} \text {. }
$$

For

$$
\mathrm{DP}_{\text {min-call out }}=\mathrm{e}^{-r T} \mathrm{E}_{Q}\left(\left(S_{T}-K\right)^{+} \mathbf{1}_{\left\{\tau^{S}>T\right\}}\right),
$$

we can get the result from the relationship

$$
\mathrm{DP}_{\text {min-call out }}=\mathrm{e}^{-r T} \mathrm{E}_{Q}\left(\left(S_{T}-K\right)^{+}\right)-\mathrm{DP}_{\text {min-call in }} .
$$

Furthermore, if we set

$$
\tilde{\tau}^{S}=\tau_{1}^{S} \vee \tau_{2}^{S}
$$

we can define another type of Parisian option by $\tilde{\tau}^{Y}$ :

$$
\mathrm{DP}_{\text {max-call in }}=\mathrm{e}^{-r T} \mathrm{E}_{Q}\left(\left(S_{T}-K\right)^{+} \mathbf{1}_{\left\{\tilde{\tau} S_{T}\right\}}\right) .
$$

In order to get its pricing formula, we should use the following relationship:

$$
\mathbf{1}_{\left\{\tilde{\tau}^{S}<T\right\}}=\mathbf{1}_{\left\{\tau_{1}^{S}<T\right\}}+\mathbf{1}_{\left\{\tau_{2}^{S}<T\right\}}-\mathbf{1}_{\left\{\tau^{S}<T\right\}} .
$$

We therefore have

$$
\mathrm{DP}_{\text {max-call in }}=\mathrm{DP}_{\text {up-in call }}+\mathrm{P}_{\text {down-in call }}-\mathrm{DP}_{\text {min-call in }} .
$$

Similarly, from

$$
\mathrm{DP}_{\text {max-call out }}=\mathrm{e}^{-r T} \mathrm{E}_{Q}\left(\left(S_{T}-K\right)^{+}\right)-\mathrm{DP}_{\max -\text { call in }},
$$

we can work out $\mathrm{DP}_{\text {max-call out }}$.

\section{Appendix A. Proof of Theorem 3}

Let $T$ be the final time. According to the definition of $\Psi(x)$, we have

$$
\Psi(x)=2 \sqrt{\pi} x \mathcal{N}(\sqrt{2} x)-\sqrt{\pi} x+\mathrm{e}^{-x^{2}}=\sqrt{\pi} x-\sqrt{\pi} x \operatorname{Erfc}(x)+\mathrm{e}^{-x^{2}} .
$$

It is not difficult to show that

$$
\mathrm{E}\left(\exp \left\{-\beta \tau^{W^{\mu}}\right\}\right)=\mathrm{E}\left(\int_{0}^{\infty} \beta \mathrm{e}^{-\beta T} \mathbf{1}_{\left\{\tau^{W^{\mu}}<T\right\}} \mathrm{d} T\right) .
$$


By Girsanov's theorem, this is equal to

$$
\int_{0}^{\infty} \beta \exp \left\{-\left(\beta+\frac{\mu^{2}}{2}\right) T-\mu x_{0}\right\} \mathrm{E}\left(\mathrm{e}^{\mu W_{T}} \mathbf{1}_{\left\{\tau^{W}<T\right\}}\right) \mathrm{d} T .
$$

Setting $\gamma=\beta+\frac{1}{2} \mu^{2}$ gives

$$
\begin{aligned}
\mathrm{E}\left(\exp \left\{-\beta \tau^{W^{\mu}}\right\}\right) & =\int_{0}^{\infty}\left(\gamma-\frac{1}{2} \mu^{2}\right) \mathrm{e}^{-\gamma T-\mu x_{0}} \mathrm{E}\left(\mathrm{e}^{\mu W_{T}} \mathbf{1}_{\left\{\tau^{W}<T\right\}}\right) \mathrm{d} T \\
& =\frac{\gamma-\mu^{2} / 2}{\gamma} \mathrm{e}^{-\mu x_{0}} \mathrm{E}\left(\mathrm{e}^{\left.\mu W_{\tilde{T}} \mathbf{1}_{\left\{\tau^{W}<\tilde{T}\right\}}\right),}\right.
\end{aligned}
$$

where $\tilde{T}$ is a random variable with an exponential distribution of parameter $\gamma$ which is independent of $W_{t}$. Therefore, we have

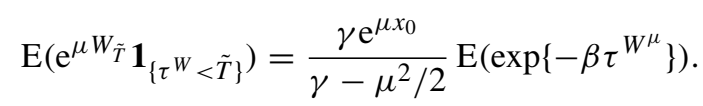

In order to invert the above moment generating function, we first need to invert the following expressions:

$$
\begin{aligned}
& \frac{\mu}{\gamma-\mu^{2} / 2}=\int_{0}^{\infty} \mathrm{e}^{\mu x} \mathrm{e}^{-\sqrt{2 \gamma} x} \mathrm{~d} x-\int_{-\infty}^{0} \mathrm{e}^{\mu x} \mathrm{e}^{\sqrt{2 \gamma} x} \mathrm{~d} x, \\
& \frac{1}{\gamma-\mu^{2} / 2}=\int_{0}^{\infty} \mathrm{e}^{\mu x} \frac{1}{\sqrt{2 \gamma}} \mathrm{e}^{-\sqrt{2 \gamma} x} \mathrm{~d} x+\int_{-\infty}^{0} \mathrm{e}^{\mu x} \frac{1}{\sqrt{2 \gamma}} \mathrm{e}^{\sqrt{2 \gamma} x} \mathrm{~d} x \text {, } \\
& \mathrm{e}^{d_{1} \mu^{2} / 2}=\int_{-\infty}^{\infty} \mathrm{e}^{\mu x} \frac{1}{\sqrt{2 \pi d_{1}}} \mathrm{e}^{-x^{2} / 2 d_{1}} \mathrm{~d} x \\
& 1-\sqrt{\frac{d_{i}}{2} \pi} \mu \mathrm{e}^{d_{i} \mu^{2} / 2} \operatorname{Erfc}\left(\sqrt{\frac{d_{i}}{2}} \mu\right)=\int_{-\infty}^{0} \mathrm{e}^{\mu x} \frac{-x}{d_{i}} \mathrm{e}^{-x^{2} / 2 d_{i}} \mathrm{~d} x .
\end{aligned}
$$

Therefore, the inversion of $\mu \mathrm{e}^{d_{1} \mu^{2} / 2} /\left(\gamma-\mu^{2} / 2\right)$ is

$$
\begin{gathered}
\int_{0}^{\infty} \mathrm{e}^{-\sqrt{2 \gamma} y} \frac{1}{\sqrt{2 \pi d_{1}}} \exp \left\{-\frac{(x-y)^{2}}{2 d_{1}}\right\} \mathrm{d} y-\int_{-\infty}^{0} \mathrm{e}^{\sqrt{2 \gamma} y} \frac{1}{\sqrt{2 \pi d_{1}}} \exp \left\{-\frac{(x-y)^{2}}{2 d_{1}}\right\} \mathrm{d} y \\
=\mathrm{e}^{\gamma d_{1}}\left\{\mathrm{e}^{-\sqrt{2 \gamma} x} \mathcal{N}\left(\frac{x}{\sqrt{d_{1}}}-\sqrt{2 \gamma d_{1}}\right)-\mathrm{e}^{\sqrt{2 \gamma} x} \mathcal{N}\left(-\frac{x}{\sqrt{d_{1}}}-\sqrt{2 \gamma d_{1}}\right)\right\} .
\end{gathered}
$$

The inversion of $1-\sqrt{d_{i} \pi / 2} \mu \mathrm{e}^{d_{i} \mu^{2} / 2} \operatorname{Erfc}\left(\sqrt{d_{i} \mu / 2}\right) /\left(\gamma-\mu^{2} / 2\right)$ is given below.

For $x \geq 0$,

$$
\int_{-\infty}^{0} \frac{-y}{d_{i}} \mathrm{e}^{-y^{2} / 2 d_{i}} \frac{1}{\sqrt{2 \gamma}} \mathrm{e}^{-\sqrt{2 \gamma}(x-y)} \mathrm{d} y=\frac{\mathrm{e}^{-\sqrt{2 \gamma} x}}{\sqrt{2 \gamma}}-\mathrm{e}^{\gamma d_{i}-\sqrt{2 \gamma} x} \sqrt{2 \pi d_{i}} \mathcal{N}\left(-\sqrt{2 \gamma d_{i}}\right) .
$$

For $x<0$,

$$
\begin{gathered}
\int_{-\infty}^{x} \frac{-y}{d_{i}} \mathrm{e}^{-y^{2} / 2 d_{i}} \frac{1}{\sqrt{2 \gamma}} \mathrm{e}^{-\sqrt{2 \gamma}(x-y)} \mathrm{d} y+\int_{x}^{0} \frac{-y}{d_{i}} \mathrm{e}^{-y^{2} / 2 d_{i}} \frac{1}{\sqrt{2 \gamma}} \mathrm{e}^{\sqrt{2 \gamma}(x-y)} \mathrm{d} y \\
=\frac{\mathrm{e}^{\sqrt{2 \gamma} x}}{\sqrt{2 \gamma}}-\mathrm{e}^{\gamma d_{i}-\sqrt{2 \gamma} x} \sqrt{2 \pi d_{i}} \mathcal{N}\left(\frac{x}{\sqrt{d_{i}}}-\sqrt{2 \gamma d_{i}}\right) \\
+\mathrm{e}^{\gamma d_{i}+\sqrt{2 \gamma} x} \sqrt{2 \pi d_{i}}\left\{\mathcal{N}\left(\sqrt{2 \gamma d_{i}}\right)-\mathcal{N}\left(\frac{x}{\sqrt{d_{i}}}+\sqrt{2 \gamma d_{i}}\right)\right\} .
\end{gathered}
$$

Consequently, we can get Theorem 3 . 


\section{Acknowledgement}

We would like to thank the anonymous referee for his/her useful comments and suggestions.

\section{References}

[1] Bertoin, J. (1997). Lévy Processes (Camb. Tracts Math. 121). Cambridge University Press.

[2] Borodin, A. N. and SAlminen, P. (1996). Handbook of Brownian Motion-Facts and Formulae. Birkhäuser, Basel.

[3] Breiman, L. (1968). Probability. Addison-Wesley, Reading, MA.

[4] Chesney, M., Jeanblanc-Picque, M. And Yor, M. (1997). Brownian excursions and Parisian barrier options. Adv. Appl. Prob. 29, 165-184.

[5] Chung, K. L. (1976). Excursions in Brownian motion. Ark. Math. 14, 155-177.

[6] Dassios, A. and Wu, S. (2010). Two-sided Parisian option with single barrier. Finance Stoch. 14, $473-494$.

[7] Davis, M. H. A. (1994). Markov Models and Optimization. Chapman and Hall, London.

[8] Labart, C. And Lelong, J. (2005). Pricing Parisian options. Tech. Rep., ENPC. Available at http:// cermics.enpc.fr/reports/CERMICS-2005/CERMICS-2005-294.pdf.

[9] Revuz, D. ANd Yor, M. (1999). Continuous Martingales and Brownian Motion, 3rd edn. Springer, Berlin.

[10] Rogers, L. C. G. (1989). A guided tour through excursions. Bull. London Math. Soc. 21, 305-341.

[11] Rogers, L. C. G. And Williams, D. (1987). Diffusions, Markov Processes and Martingales, Vol. 2. John Wiley, New York.

[12] Rogers, L. C. G. and Williams, D. (1994). Diffusions, Markov Processes and Martingales, Vol. 1, 2 nd edn. John Wiley, Chichester.

[13] Schröder, M. (2003). Brownian excursions and Parisian barrier options: a note. J. Appl. Prob. 40, $855-864$.

[14] Yor, M. (1997). Some Aspects of Brownian Motion. Part II. Birkhäuser, Basel. 